\title{
On Ground Word Problem of Term Equation Systems
}

\author{
Sándor Vágvölgyi* \\ To the memory of my teacher and colleague Ferenc Gécseg
}

\begin{abstract}
We give semi-decision procedures for the ground word problem of variable preserving term equation systems and term equation systems. They are natural improvements of two well known trivial semi-decision procedures. We show the correctness of our procedures.
\end{abstract}

Keywords: term equation systems; ground word problem; Knuth-Bendix completion procedure; ground term rewriting systems

\section{Introduction}

A term equation $l \approx r$ is called variable preserving if the same variables occur in the left-hand side $l$ as in the right-hand side $r$. A term equation system (TES) $E$ is called variable preserving if all of its equations are variable preserving. The ground word problem is undecidable even for variable-preserving TESs, see Example 4.1 .4 on page 60 in [1]. We recall the well known trivial semi-decision procedure PRO1 for the ground word problem of variable preserving TESs and its straightforward generalization, the trivial semi-decision procedure PRO2 for the ground word problem of TESs.

On the basis of PRO1, we give a semi-decision procedure PRO3 for the ground word problem of variable preserving TESs. Given a TES $E$ and ground terms $p, q$ over the ranked alphabet $\Sigma$, procedure $P R O 3$ constructs the ground TESs (GTESs) $P_{i}$ and $Q_{i}, i \geq 1$ such that

(a) $P_{i} \cup Q_{i} \subseteq \leftrightarrow_{E}^{*}$ for $i \geq 1$.

Condition (a) ensures that the congruence closure of $P_{i} \cup Q_{i}$ is a subset of $\leftrightarrow_{E}^{*}$. Procedure $P R O 3$ outputs an answer and halts if and only if

(b) there is a $j \geq 1$ such that

$p \leftrightarrow{ }_{P_{j}}^{*} \cup Q_{j} q$ or

$\leftrightarrow_{P_{j}}^{*} \cap\left(\{p\} \times T_{\Sigma}\right)=\leftrightarrow_{E}^{*} \cap\left(\{p\} \times T_{\Sigma}\right)$ or

\footnotetext{
*Department of Foundations of Computer Science, University of Szeged, Árpád tér 2, H-6720 Szeged, Hungary. E-mail: vagvolgy@inf.u-szeged.hu
} 
$\leftrightarrow_{Q_{j}}^{*} \cap\left(\{q\} \times T_{\Sigma}\right)=\leftrightarrow_{E}^{*} \cap\left(\{q\} \times T_{\Sigma}\right)$.

Condition (b) says that we have a proof of $p \leftrightarrow_{E}^{*} q$, or the intersection of $\leftrightarrow_{P_{j}}^{*}$ with $\left(\{p\} \times T_{\Sigma}\right)$ is equal to that of $\leftrightarrow_{E}^{*}$, or the intersection of $\leftrightarrow_{Q_{j}}^{*}$ with $\left(\{q\} \times T_{\Sigma}\right)$ is equal to that of $\leftrightarrow_{E}^{*}$. Assume that (b) holds. If $p \leftrightarrow_{P_{j} \cup Q_{j}}^{*} q$ holds, PRO3 outputs 'yes', and halts. Otherwise, if

- the intersection of $\leftrightarrow_{P_{j}}^{*}$ with $\left(\{p\} \times T_{\Sigma}\right)$ is equal to that of $\leftrightarrow_{E}^{*}$, or

- the intersection of $\leftrightarrow_{Q_{j}}^{*}$ with $\left(\{q\} \times T_{\Sigma}\right)$ is equal to that of $\leftrightarrow_{E}^{*}$,

then $p \leftrightarrow{ }_{E}^{*} q$ does not hold either. Hence semi-decision procedure PRO3 outputs 'no' and halts.

Procedure PRO 3 constructs the ground TESs (GTESs) $P_{i}$ and $Q_{i}, i \geq 1$ in the following way. We put a ground instance $l^{\prime} \approx r^{\prime}$ of an equation $l \approx r$ of $E \cup E^{-1}$ in $P_{1}$ if $l^{\prime}$ is a subterm of $p$. Then we iterate the following computation items.

- We convert the GTES $P_{i}$ into an equivalent reduced ground term rewrite system $R_{i}$ applying Snyder's fast ground completion algorithm [19].

- We define the GTES $P_{i+1}$ from the reduced ground term rewrite system $R_{i}$ by adding all ground instances $l \approx r$ of equations in $E \cup E^{-1}$ such that

$-l \approx r$ is not in $\leftrightarrow_{P_{i}}^{*}$ and that

- there exists a term $s$ such that the conversion $p \leftrightarrow_{P_{i}}^{*} s$ can be continued applying $l \approx r$ to $s$. If $P_{i+1}=R_{i}$, then we let $R_{i+1}=R_{i}$, and hence $R_{i}=P_{j}=R_{j}$ holds for $j \geq i+1$.

Here we consider both the reduced ground term rewrite system $R_{i}$ and the GTES $P_{i+1}$ as subsets of $T_{\Sigma} \times T_{\Sigma}$. Furthermore, we consider a ground instance of an equation in $E \cup E^{-1}$ as an element of $T_{\Sigma} \times T_{\Sigma}$.

We define the GTES $Q_{i}$ symmetrically to $P_{i}$ for $i \geq 1$.

Procedure $P R O 3$ computes in the following way. For each $i=1,2, \ldots$,

- if $p \leftrightarrow P_{P_{i} \cup Q_{i}}^{*} q$, then we output the answer 'yes' and halt;

- otherwise, if $i \geq 2$ and we did not add ground instances of equations in $E \cup E^{-1}$ to the reduced ground term rewrite system $R_{P_{i-1}}$, equivalent to $P_{i-1}$, or to the reduced ground term rewrite system $R_{Q_{i}}$, equivalent to $Q_{i-1}$, in the previous iteration step, then we output the answer 'no' and halt.

Assume that $p \leftrightarrow_{E}^{*} q$. Then, at some step during the run of procedure PRO3, $p \leftrightarrow{ }_{P \cup Q}^{*} q$ becomes true, and procedure PRO3 outputs 'yes' and halt. If $p \leftrightarrow_{E}^{*} q$ does not hold, then procedure $P R O 3$ either outputs 'no' and halts or runs forever.

We give a semi-decision procedure $\mathrm{PRO}_{4}$ for the ground word problem of TESs. We obtain it generalizing PRO3 taking into account PRO2. The main difference is the following. We define $P_{i+1}$ from $R_{i}$ by adding all ground instances $l^{\prime} \approx r^{\prime}$ of the equations $l \approx r$ in $E \cup E^{-1}$ such that

- $l^{\prime} \approx r^{\prime}$ is not in $\leftrightarrow_{P_{i}}^{*}$, that

- there exists a term $s$ such that a conversion $p \leftrightarrow P_{P_{i}}^{*} s$ can be continued applying $l \approx r$ to $s$, and that

- we substitute some finitely many ground terms depending on $i, R_{i}$, and $p$, for those variables in $r$ that do not appear in $l$.

We modify the halting condition of the proceedure so that it stops if we did not add ground instances of equations in $E \cup E^{-1}$ to $P_{i}$ or $Q_{i}$ in two successive iteration 
steps. We need two successive steps rather than one. Because, in general, the heights of the substituted terms becomes larger in each step. If we do not add ground equations to $P_{i}$ in a step, then in the next step we still may add ground equations to $P_{i}$.

Procedures $\mathrm{PRO}_{3}$ and $\mathrm{PRO}_{4}$ compute in a different way than all versions of the Knuth-Bendix completion procedure. To some instances of the ground word problem of a TES E, procedures $\mathrm{PRO}_{3}$ and $\mathrm{PRO}_{4}$ give an answer sooner than all versions of the Knuth-Bendix completion procedure or it is open whether some version of the Knuth-Bendix completion procedure gives an answer at all. Consequently, they may compute efficiently for some instances of the ground word problem of a TES $E$, when the various versions of the Knuth-Bendix completion procedure does not give an answer to the ground word problem of a TES $E$ at all or at least not in a reasonable time. However, it is still open in which cases are $\mathrm{PRO}_{3}$ and $\mathrm{PRO}_{4}$ really efficient.

In Section 2, we present a brief review of the notions, notations, and preliminary results used in the paper. In Section 3 we introduce and study the concept of reading-up reachability for reduced ground term rewriting systems. In Section 4 we present the procedures PRO1 and PRO2. In Section 5, we present the procedure $P R O 3$, and show its correctness. We give examples when procedure $P R O 3$ is more efficient than procedure PRO1. In Section 6, we present the procedure PRO4, and show its correctness. In Section 7, we compare procedures PRO3 and PRO4 with the basic Knuth-Bendix completion procedure (see Section 7.1 in [1]), an improved version of the Knuth-Bendix completion procedure described by a set of inference rules (see Section 7.2 in [1]), the goal-directed completion procedure based on SOUR graphs [13, 14], and the unfailing Knuth-Bendix completion procedure [2]. In Section 8, we sum up our results, and explain the applicability of procedures $\mathrm{PRO} 3$ and $\mathrm{PRO}_{4}$.

\section{Preliminaries}

In this section we present a brief review of the notions, notations and preliminary results used in the paper. For all unexplained notions and notation see [1].

Relations. Let $\rho$ be an equivalence relation on $A$. Then for every $a \in A$, we denote by $a / \rho$ the $\rho$-class containing $a$, i.e. $a / \rho=\{b \mid a \rho b\}$. For each $B \subseteq A$, let $B / \rho=\{b / \rho \mid b \in B\}$.

\subsection{Abstract Reduction Systems}

An abstract reduction system is a pair $(A, \rightarrow)$, where the reduction $\rightarrow$ is a binary relation on the set $A . \rightarrow^{-1}, \leftrightarrow, \rightarrow^{*}$, and $\leftrightarrow^{*}$ denote the inverse, the symmetric closure, the reflexive transitive closure, and the reflexive transitive symmetric closure of the binary relation $\rightarrow$, respectively.

- $x \in A$ is reducible if there is $y$ such that $x \rightarrow y$.

- $x \in A$ is irreducible if it is not reducible. 
- $y \in A$ is a normal form of $x \in A$ if $x \rightarrow^{*} y$ and $y$ is irreducible. If $x \in A$ has a unique normal form, the latter is denoted by $x \downarrow$.

- $y \in A$ is a descendant of $x \in A$ if $x \rightarrow^{*} y$.

- $x \in A$ and $y \in A$ are joinable if there is a $z$ such that $x \rightarrow^{*} z \leftarrow^{*} y$, in which case we write $x \downarrow y$.

The reduction $\rightarrow$ is called

- confluent if for all $x, y_{1}, y_{2} \in A$, if $y_{1} \leftarrow^{*} x \rightarrow^{*} y_{2}$, then $y_{1} \downarrow y_{2}$;

- locally confluent if for all $x, y_{1}, y_{2} \in A$, if $y_{1} \leftarrow x \rightarrow y_{2}$, then $y_{1} \downarrow y_{2}$;

- terminating if there is no infinite chain $x_{0} \rightarrow x_{1} \rightarrow x_{2} \rightarrow \cdots$;

- convergent if it is both confluent and terminating.

If $\rightarrow$ is convergent, then each $x \in A$ has a unique normal form [1].

Terms. A ranked alphabet $\Sigma$ is a finite set of symbols in which every element has a unique rank in the set of nonnegative integers. For each integer $m \geq 0, \Sigma_{m}$ denotes the elements of $\Sigma$ which have rank $m$.

Let $Y$ be a set of variables. The set of terms over $\Sigma$ with variables in $Y$ is denoted by $T_{\Sigma}(Y)$. The set $T_{\Sigma}(\emptyset)$ is written simply as $T_{\Sigma}$ and called the set of ground terms over $\Sigma$. We specify a countably infinite set $X=\left\{x_{1}, x_{2}, \ldots\right\}$ of variables which will be kept fixed in this paper. Moreover, we put $X_{n}=\left\{x_{1}, x_{2}, \ldots, x_{n}\right\}$, for $n \geq 0$. Hence $X_{0}=\emptyset$. For any $i \geq 1$ and $j \geq 0$, let $X_{[i, j]}=\emptyset$ if $i>j$, and let $X_{[i, j]}=\left\{x_{i}, x_{i+1}, \ldots, x_{j}\right\}$ otherwise.

For a term $t \in T_{\Sigma}(X)$, the height height $(t) \in N$ is defined by recursion:

(a) if $t \in \Sigma_{0} \cup X$, then height $(t)=0$,

(b) if $t=\sigma\left(t_{1}, \ldots, t_{m}\right)$ with $m \geq 1$ and $\sigma \in \Sigma_{m}$, then height $(t)=1+\max \left(\right.$ height $\left.\left(t_{i}\right) \mid 1 \leq i \leq m\right)$.

For each $k \geq 0, H E_{\Sigma, \leq k}(X)=\left\{t \in T_{\Sigma}(X) \mid \operatorname{height}(t) \leq k\right\}$.

Let $N$ be the set of all positive integers. $N^{*}$ stands for the free monoid generated by $N$ with empty word $\lambda$ as identity element. For each word $\alpha \in N^{*}$, length $(\alpha)$ stands for the length of $\alpha$. Consider the words $\alpha, \beta, \gamma \in N^{*}$ such that $\alpha=\beta \gamma$. Then we say that $\beta$ is a prefix of $\alpha$. Furthermore, if $\alpha \neq \beta$, then $\beta$ is a proper prefix of $\alpha$. For a term $t \in T_{\Sigma}(X)$, the set $\operatorname{Pos}(t) \subseteq N^{*}$ of positions is defined by recursion:

(i) if $t \in \Sigma_{0} \cup X$, then $\operatorname{Pos}(t)=\{\lambda\}$, and

(ii) if $t=\sigma\left(t_{1}, \ldots, t_{m}\right)$ with $m \geq 1$ and $\sigma \in \Sigma_{m}$, then $\operatorname{Pos}(t)=\{\lambda\} \cup\{i \alpha \mid 1 \leq$ $i \leq m$ and $\left.\alpha \in \operatorname{Pos}\left(t_{i}\right)\right\}$.

For each term $t \in T_{\Sigma}(X)$, size $(t)$ is the cardinality of $\operatorname{Pos}(t)$.

For each $t \in T_{\Sigma}(X)$ and $\alpha \in \operatorname{Pos}(t)$, we introduce the subterm $t / \alpha \in T_{\Sigma}(X)$ of $t$ at $\alpha$ as follows:

(a) for $t \in \Sigma_{0} \cup X, t / \lambda=t$;

(b) for $t=\sigma\left(t_{1}, \ldots, t_{m}\right)$ with $m \geq 1$ and $f \in \Sigma_{m}$, if $\alpha=\lambda$ then $t / \alpha=t$, otherwise, if $\alpha=i \beta$ with $1 \leq i \leq m$, then $t / \alpha=t_{i} / \beta$

For any $t \in T_{\Sigma}(X), \alpha \in \operatorname{Pos}(t)$, and $r \in T_{\Sigma}(X)$, we define $t[\alpha \leftarrow r] \in T_{\Sigma}(X)$. 
(i) If $\alpha=\lambda$, then $t[\alpha \leftarrow r]=r$.

(ii) If $\alpha=i \beta$, for some integer $i$, then $t=\sigma\left(t_{1}, \ldots, t_{m}\right)$ with $f \in \Sigma_{m}$ and $1 \leq i \leq m$. Then $t[\alpha \leftarrow r]=\sigma\left(t_{1}, \ldots, t_{i-1}, t_{i}[\beta \leftarrow r], t_{i+1}, \ldots, t_{m}\right)$.

For a term $t \in T_{\Sigma}(X)$, the set $s u b(t)$ of subterms of $t$ is defined as $s u b(t)=$ $\{t / \alpha \mid \alpha \in \operatorname{Pos}(t)\}$.

Given a term $t \in T_{\Sigma}\left(X_{n}\right), n \geq 0$, and terms $t_{1}, \ldots, t_{n}$, we denote by $t\left[t_{1}, \ldots, t_{n}\right]$ the term which can be obtained from $t$ by replacing each occurrence of $x_{i}$ in $t$ by $t_{i}$ for $1 \leq i \leq n$. A context is a term $u \in T_{\Sigma \cup\{\diamond\}}$, where the nullary symbol $\diamond$ appears exactly once in $u$. We denote the set of all contexts over $\Sigma$ by $C_{\Sigma}$. For a context $u$ and a term $t, u[t]$ is defined from $u$ by replacing the occurrence of $\diamond$ with $t$.

For the sake of simplicity, we may write unary terms as strings. For example, we write $f g h \#$ for the term $f(g(h(\#)))$ and $f^{3} x_{1}$ for $f\left(f\left(f\left(x_{1}\right)\right)\right)$, where $f, g, h$ are unary symbols and \# is a nullary symbol.

Algebras. Let $\Sigma$ be a ranked alphabet. A $\Sigma$ algebra is a system $\mathbf{B}=\left(B, \Sigma^{\mathbf{B}}\right)$, where $B$ is a nonempty set, called the carrier set of $\mathbf{B}$, and $\Sigma^{\mathbf{B}}=\left\{f^{\mathbf{B}} \mid f \in \Sigma\right\}$ is a $\Sigma$-indexed family of operations over $B$ such that for every $f \in \Sigma_{m}$ with $m \geq 0$, $f^{\mathbf{B}}$ is a mapping from $B^{m}$ to $B$. An equivalence relation $\rho \subseteq B \times B$ is a congruence on $\mathbf{B}$ if

$$
f^{\mathbf{B}}\left(t_{1}, \ldots, t_{m}\right) \rho f^{\mathbf{B}}\left(p_{1}, \ldots, p_{m}\right)
$$

whenever $f \in \Sigma_{m}, m \geq 0$, and $t_{i} \rho p_{i}$, for $1 \leq i \leq m$. For each $B^{\prime} \subseteq B$, let $\left[B^{\prime}\right]_{\rho}=\left\{[b]_{\rho} \mid b \in B^{\prime}\right\}$. In this paper we shall mainly deal with the algebra $\mathbf{T A}=\left(T_{\Sigma}, \Sigma\right)$ of ground terms over $\Sigma$, where for any $f \in \Sigma_{m}$ with $m \geq 0$ and $t_{1}, \ldots, t_{m} \in T_{\Sigma}$, we have

$$
f^{\mathbf{T A}}\left(t_{1}, \ldots, t_{m}\right)=f\left(t_{1}, \ldots, t_{m}\right) .
$$

We now recall the concept of a set of representatives for a congruence $\rho$ and a set of $\rho$-classes.

Definition 1. [6] Let $\rho$ be a congruence on TA and let $A$ be a set of $\rho$-classes. A set $R E P$ of ground terms is called a set of representatives for $A$ if

- $R E P \subseteq \bigcup A$,

- $\bigcup(s u b(t) \mid t \in R E P) \subseteq R E P$, and

- each class $Z \in A$ contains exactly one term $t \in R E P$.

Term equation systems. Let $\Sigma$ be a ranked alphabet. A term equation system (TES for short) $E$ over $\Sigma$ is a finite subset of $T_{\Sigma}(X) \times T_{\Sigma}(X)$. Elements $(l, r)$ of $E$ are called equations and are denoted by $l \approx r$. The reduction relation $\rightarrow_{E} \subseteq$ $T_{\Sigma}(X) \times T_{\Sigma}(X)$ is defined as follows. For any terms $s, t \in T_{\Sigma}(X), s \rightarrow_{E} t$ if there is a pair $l \approx r$ in $E$ and a context $u \in C_{\Sigma}\left(X_{1}\right)$ and a substition $\delta$ such that $s=u[\delta(l)]$ and $t=u[\delta(r)]$. When we apply an arbitrary equation $l \approx r \in E \cup E^{-1}$, we rename the variables of $l$ and $r$ such that $l \in T_{\Sigma}\left(X_{k+m}\right)$ and $r \in T_{\Sigma}\left(X_{k} \cup X_{[k+m+1, k+m+\ell]}\right)$ for some $k, m, \ell \geq 0$. 
The word problem for a TES $E$ is the problem of deciding for arbitrary $p, q \in$ $T_{\Sigma}(X)$ whether $p \leftrightarrow_{E}^{*} q$. The ground word problem for $E$ is the word problem restricted to ground terms $p$ and $q$.

For the notion of a term rewriting system (TRS), see Section 4.2 in [1]

Knuth-Bendix completion procedure. We now briefly recall the basic Knuth-Bendix completion procedure, see Section 7.1 in [1]. The basic KnuthBendix completion procedure starts with a TES $E$ and tries to find a convergent TRS $R$ that is equivalent to $E$. A reduction order $>$ is provided as an input for the procedure. Since the word problem is not decidable in general, a finite convergent TRS cannot always be obtained. In the basic Knuth-Bendix completion procedure this could be due to failure or to non-termination of completion. In the initialization phase, the basic completion procedure removes trivial identities of the form $s=s$ and tries to orient the remaining nontrivial identities. If this succeeds, then it computes all critical pairs of the TRS obtained. The terms in each critical pair $\langle s, t\rangle$ are reduced to their normal forms $\hat{s}$ and $\hat{t}$. If the normal forms are identical, then this critical pair is joinable, and nothing needs to be done for it. Otherwise, the procedure tries to orient the terms $\hat{s}$ and $\hat{t}$ into the rewrite rule $\hat{s} \rightarrow \hat{t}$ with $\hat{s}>\hat{t}$ or $\hat{t} \rightarrow \hat{s}$ with $\hat{t}>\hat{s}$. In this way the procedure orients all instances of the terms $\hat{s}$ and $\hat{t}$ as well. If this succeeds, then the new rule is added to the current rewrite system. This process is iterated until failure occurs or the rewrite system is not changed during a step of the iteration, that is, the system does not have non-joinable critical pairs.

If the basic completion procedure applied to $(E,>)$ terminates succesfully with output $R$, then $R$ is a finite convergent TRS that is equivalent to $E$. In this case, $R$ yields a decision procedure for the word problem for $E$. If the basic completion procedure applied to $(E,>)$ does not terminate, then it outputs an infinite convergent TRS that is equivalent to $E$. In this case, the completion procedure can be used as a semidecision procedure for the word problem for $E$.

Assume that we want to decide for given terms $p, q \in T_{\Sigma}(X)$, whether $p \leftrightarrow_{E}^{*} q$ holds. We call the pair $(p, q)$ the goal. The basic Knuth-Bendix completion procedure is independent of the goal. Hence, if $p \leftrightarrow_{E}^{*} q$ does not hold, and the set $E$ of equations has no finite convergent system, then the basic Knuth-Bendix completion will run forever. In the light of this observation, Lynch and Strogova $[13,14]$ presented a goal-directed completion procedure based on SOUR graphs. Similarly to the basic Knuth-Bendix completion procedure, the goal-directed completion procedure uses a reduction order $>$. Unlike the basic Knuth-Bendix completion procedure, it uses some inference rules. The main difference, described in an intuitive simplified way, is the following. Along the completion procedure, we try to construct a rewrite system $R$ and a conversion

$$
p=r_{1} \underset{R}{\overleftrightarrow{R}} r_{2} \underset{R}{\leftrightarrow} \cdots \underset{R}{\leftrightarrow} r_{n}=q, n \geq 1
$$

in a nondeterministic way. We compute and orient critical pairs and control the completion process keeping in our mind that the rules of $R$ should be applicable along a conversion (1). When orienting the equations into rules along the comple- 
tion process, we do not put a rule in $R$ if it is not applicable along a conversion (1). If we do not find a conversion (1), the goal-directed completion procedure detects that $(p, q) \notin \leftrightarrow_{E}^{*}$, outputs 'no' and halts. Consider the following example. Let ranked alphabet $\Sigma$ consist of the unary symbols $f, g$ and the nullary symbols $\$$, \#. Consider the variable preserving TES $E=\{f f x \approx g f x\}$. We raise the problem whether $\$ \leftrightarrow_{E}^{*} \#$. The basic Knuth-Bendix completion procedure runs forever on this example [13]. Along the goal oriented completion procedure, we find no rewrite rule such that it is applicable along a conversion $\$=r_{1} \leftrightarrow_{R} r_{2} \leftrightarrow_{R} \cdots \leftrightarrow_{R} r_{n}=\#$, $n \geq 1$. Therefore, the goal-directed completion procedure detects that $(\$, \#) \notin \leftrightarrow_{E}^{*}$, outputs 'no', and halts [13].

We now adopt a more detailed description of the goal-directed completion procedure. [14] The goal-directed completion procedure uses a reduction order $>$ and computes critical pairs equipped with equational and ordering constraints, and constructs a graph. "The goal-directed completion procedure has two phases. The first phase is the compilation phase. In this phase, all the edges and the recursive constraints labelling each edge are created. This phase also takes into account the goal to be solved. Importantly, this phase takes only polynomial time, because there are only polynomially many edges in the graph. The result of this phase is a constrained tree automaton representing a schematized version of the completed system, and a set of constraints representing potential solutions to the goal. The constraints that are generated are the equational constraints representing the unification problems, and ordering constraints arising from the critical pair inferences.

The second phase is the goal solving (or constraint solving) phase. In this phase, the potential solutions to the goal are solved in order to determine whether they are actual solutions of the goal. This phase can take infinitely long, since the constraints are recursive. Step by step a constraint is rolled back, based on which edges it is created from, and the equational and ordering constraints are solved along the way. In some cases, the ordering constraints cause the recursion to halt, and therefore the constraints are completely solved. The procedure is truly goal oriented, because only a polynomial amount of time is spent compiling the set of equations. The rest of the time is spent working backwards from the goal to solve the constraints. If the procedure is examined more closely, we see that the second phase of the procedure is exactly a backwards process of completion. A schematization of an equation in the completed system is applied to the goal, step by step until it rewrites to an identity. At the same time, the schematized equation that is selected is worked backwards until we reach the original equations from which it is formed." [14]

See Section 7.2 in [1] for an improved version of the Knuth-Bendix completion procedure described by a set of inference rules. A detailed description of the unfailing Knuth-Bendix completion procedure can be found in [2].

Ground term equation systems and rewriting systems. A ground term equation system (GTES) $E$ over a ranked alphabet $\Sigma$ is a finite binary relation on $T_{\Sigma}$. Elements $(l, r)$ of $E$ are called equations and are denoted by $l \approx r$. The reduction relation $\rightarrow_{E} \subseteq T_{\Sigma}(X) \times T_{\Sigma}(X)$ is defined as follows. For any ground terms $s, t \in T_{\Sigma}, s \rightarrow_{E} t$ if there is a pair $l \approx r$ in $E$ and a context $u \in C_{\Sigma}\left(X_{1}\right)$ 
such that $s=u[l]$ and $t=u[r]$. It is well known that the relation $\leftrightarrow_{E}^{*}$ is a congruence on the term algebra TA [18]. We call $\leftrightarrow_{E}^{*}$ the congruence induced by $E$. The size of $E$ is defined as the number of occurrences of symbols in the set. $\operatorname{sub}(E)=\left\{\operatorname{sub}(l) \mid l \approx r \in E \cup E^{-1}\right\}$. Clearly, $\leftrightarrow_{E}^{*} \cap(\operatorname{sub}(E) \times \operatorname{sub}(E))$ is an equivalence relation on $\operatorname{sub}(E)$. The word problem for a GTES $E$ is the problem of deciding for arbitrary $p, q \in T_{\Sigma}$ whether $p \leftrightarrow_{E}^{*} q$.

A ground term rewrite system (GTRS) over a ranked alphabet $\Sigma$ is a finite subset $R$ of $T_{\Sigma} \times T_{\Sigma}$. The elements of $R$ are called rules and a rule $(l, r) \in R$ is written in the form $l \rightarrow r$ as well. Moreover, we say that $l$ is the left-hand side and $r$ is the right-hand side of the rule $l \rightarrow r$. $\operatorname{lh} s(R)=\{l \mid l \rightarrow r \in R\}$, $\operatorname{rhs}(R)=\{r \mid l \rightarrow r \in R\} . \operatorname{sub}(R)=\{\operatorname{sub}(l) \mid l \in \operatorname{lhs}(R)\} \cup\{\operatorname{sub}(r) \mid r \in \operatorname{lhs}(R)\}$.

The reduction relation $\rightarrow_{R} \subseteq T_{\Sigma}(X) \times T_{\Sigma}(X)$ is defined as follows. For any ground terms $s, t \in T_{\Sigma}, s \rightarrow_{R} t$ if there is a pair $l \approx r$ in $E$ and a context $u \in C_{\Sigma}\left(X_{1}\right)$ such that $s=u[l]$ and $t=u[r]$. Here we say that $R$ rewrites $s$ to $t$ applying the rule $l \rightarrow r$. A GTRS $R$ is equivalent to a GTRS $E$, if $\leftrightarrow_{R}^{*}=\leftrightarrow_{E}^{*}$ holds.

$\operatorname{IRR}(R)$ denotes the set of all ground terms irreducible by $R$. A GTRS $R$ is reduced if for every rule $u \rightarrow v$ in $R, u$ is irreducible with respect to $R-\{u \rightarrow v\}$, and $v$ is irreducible with respect to $R$. For a reduced GTRS $R, \operatorname{IRR}(R) \cap \operatorname{sub}(R)=$ $\operatorname{sub}(R)-l h s(R)$, and $\operatorname{sub}(R)-l h s(R)$ is a set of representatives for $\operatorname{sub}(R) / \leftrightarrow_{R}^{*}$, see Theorem 3.14 on page 162 in [17].

We say that a GTRS $R$ is confluent, locally confluent, terminating, or convergent, if $\rightarrow_{R}$ has the corresponding property.

We recall the following important result.

Proposition 1. [19] Any reduced GTRS $R$ is convergent.

Proposition 2. For a reduced GTRS $R$, one can reduce a ground term $t \in T_{\Sigma}$ to its normal form in linear time of size $(t)$. We traverse the term $t$ in postorder. When visiting a position $\alpha$, we reduce the subterm $t / \alpha$ of $t$ at $\alpha$ to is normal form $t / \alpha \downarrow_{R}$.

We say that a GTRS $R$ is equivalent to a GTES $E$ if $\leftrightarrow_{R}^{*}=\leftrightarrow_{E}^{*}$.

Proposition 3. [19] For a GTES E one can effectively construct an equivalent reduced GTRS R in $O(n \log n)$ time. Here $n$ is the size of $E$.

Proof. We briefly recall Snyder's [19] fast ground completion algorithm. We run a congruence closure algorithm for $E$ over the subterm graph of $E[4,15]$. In this way we get the representation of the equivalence relation $\leftrightarrow_{E}^{*} \cap(\operatorname{sub}(E) \times \operatorname{sub}(E))$. We compute a set $R E P$ of representatives for $\operatorname{sub}(E) / \leftrightarrow_{E}^{*}$. Then we construct a reduced GTRS $R$ over $\Sigma$ as follows. We put the rewrite rule $l \rightarrow r$ in $R$ if

- $l=f\left(p_{1}, \ldots, p_{m}\right)$ for some $f \in \Sigma_{m}, m \geq 0$, and $p_{1}, \ldots, p_{m} \in R E P$,

- $r \in R E P$,

$\bullet l \neq r$ and $l \leftrightarrow_{E}^{*} r$.

We can decide the word problem of a GTES $E$ applying a congruence closure algorithm $[4,15]$ for the GTES $E_{1}=E \cup\{p \approx p, q \approx q\}$ and then examine whether 
$p, q$ are in the same class of the equivalence relation $\leftrightarrow_{E_{1}}^{*} \cap\left(\operatorname{sub}\left(E_{1}\right) \times \operatorname{sub}\left(E_{1}\right)\right)$. Assume that we want to solve the word problem of a fixed GTES $E$ for varying terms $p, q$. Then we compute a convergent GTRS over $\Sigma$ equivalent to $E[8,14,16,19]$. We compute $p \downarrow_{R}$ and $q \downarrow_{R}$, and compare them. If $p \downarrow_{R}=q \downarrow_{R}$, then $p \leftrightarrow_{E}^{*} q$. Otherwise, $(p, q) \notin \leftrightarrow_{E}^{*}$. By Proposition 2, we can decide the word problem of $E$ in linear time. We can also extend the signature. We introduce constants for the equivalence classes of $\leftrightarrow_{E}^{*} \cap(\operatorname{sub}(E) \times s u b(E))$. Then we can construct in $O(n \log n)$ time a reduced GTRS over the extended signature such that $p \downarrow_{R}=q \downarrow_{R}$ if and only if $p \leftrightarrow_{E}^{*} q$. By Proposition 2, we can decide the word problem of $E$ in linear time. Finally, assume that we want to solve the word problem of a fixed GTES $E$ for a fixed term $p$ and varying term $q$. Then we can construct in $O(n \log n)$ time a deterministic tree automaton recognizing the $\leftrightarrow_{E}^{*}$-class of $p$ [17].

For other completion algorithms on GTRSs see $[5,16]$. For further results on GTRSs see [18]. Proposition 1 and Proposition 3 imply the following well known result.

Proposition 4. [19] For a GTES E and ground terms p, q, one can decide whether $p \leftrightarrow{ }_{E}^{*} q$.

\section{Reachability starting from a term attached to a context}

Let $R$ be a reduced GTRS over $\Sigma$ and $s, t \in I R R(R)$. We say that $R$ reaches $t$ starting from $s$ attached to some context, if there is a $u \in C_{\Sigma}$ such that $u[s] \rightarrow_{R}^{*} t$. Let $R A C(s)$ denote the set of all terms $t \in I R R(R)$ which are reachable by $R$ starting from $s$ attached to some context.

Example 1. Let $\Sigma=\Sigma_{0} \cup \Sigma_{1}, \Sigma_{0}=\{0,1\}$, and $\Sigma_{2}=\{f\}$. Let GTRS $R$ consist of the equations $f(0,0) \rightarrow 0$ and $f(0,1) \rightarrow 1$. Clearly $R$ is reduced. Then each element of $\operatorname{IRR}(R)$ containing 0 is in $R A C(0)$. For example, $f(f(1,0), 1) \in R A C(0)$, because $f(f(1, \diamond), 1) \in C_{\Sigma}$ and

$$
f(f(1, \diamond), 1)[0]=f(f(1,0), 1) \rightarrow_{R}^{*} f(f(1,0), 1) .
$$

Furthermore, $1 \in R A C(0)$, because

$$
f(\diamond, 1)[0]=f(0,1) \rightarrow_{R} 1 .
$$

Thus each element of $\operatorname{IRR}(R)$ containing 1 is in $R A C(0)$. Consequently, $\operatorname{IRR}(R)=$ $R A C(0)$.

Lemma 1. Let $R$ be a reduced GTRS over $\Sigma$. For any $s \in \operatorname{sub}(R)-\operatorname{lh} s(R)$, we can effectively compute $R A C(s) \cap(\operatorname{sub}(R)-\operatorname{lh} s(R))$.

Proof. Let $R A C_{0}=\{s\}$. For each $i \geq 0$, let $R A C_{i+1}$ consists of all elements $t$, where

- $t \in R A C_{i}$ or

- $t \in \operatorname{sub}(R)-\operatorname{lhs}(R)$ and there is a rule $f\left(t_{1}, \ldots, t_{m}\right) \rightarrow t$ in $R$ for some $f \in \Sigma_{m}, t_{1}, \ldots, t_{m} \in \operatorname{sub}(R)-\operatorname{lhs}(R)$, such that $t_{j} \in R A C_{i}$ for some $1 \leq j \leq m$, or 
- $t \in \operatorname{sub}(R)-l h s(R)$ and $t=f\left(t_{1}, \ldots, t_{m}\right)$ for some $f \in \Sigma_{m}, t_{1}, \ldots, t_{m} \in$ $\operatorname{sub}(R)-l h s(R)$, and $t_{j} \in R A C_{i}$ for some $1 \leq j \leq m$. Then

$$
R A C_{i} \subseteq R A C_{i+1} \subseteq R A C(s) \cap(\operatorname{sub}(R)-l h s(R)) \text { for } i \geq 0 .
$$

Hence there is an integer $0 \leq \ell \leq \operatorname{card}(\operatorname{sub}(R)-\operatorname{lhs}(R))$ such that $R A C_{\ell}=R A C_{\ell+1}$. Then

$$
R A C_{\ell}=R A C_{\ell+k} \text { for } k \geq 1 .
$$

Hence

$$
R A C_{\ell} \subseteq R A C(s) \cap(\operatorname{sub}(R)-\operatorname{lhs}(R)) .
$$

To show the reverse inclusion, we need the following.

Claim 1. For any $u \in C_{\Sigma}$ of height $n \geq 0$ and $t \in \operatorname{sub}(R)-\operatorname{lhs}(R)$, if $u(s) \rightarrow_{R}^{*} t$, then $t \in R A C_{n}$.

Proof. By induction on $n$.

By (2), (3), and Claim 1, $R A C(s) \cap(\operatorname{sub}(R)-l h s(R)) \subseteq R A C_{\ell}$. By (4),

$$
R A C(s) \cap(\operatorname{sub}(R)-\operatorname{lh} s(R))=R A C_{\ell} .
$$

We compute the sets $R A C_{0}, R A C_{1}, \ldots, R_{\operatorname{card}(\operatorname{sub}(R)-l h s(R))}$. In this way we obtain the integer $\ell$ and $R A C(s) \cap(\operatorname{sub}(R)-l h s(R))$.

Lemma 2. For any reduced GTRS $R$ and $s, t \in I R R(R), R$ reaches $t$ starting from $s$ attached to some context if and only if

(i) $t=u[s]$ for some $u \in C_{\Sigma}$ or

(ii) $s \in(\operatorname{sub}(R)-l h s(R))$, and there are $u \in C_{\Sigma}$ and $r \in \operatorname{rhs}(R)$ such that $t=u[r]$ and $R$ reaches $r$ starting from $s$ attached to some context.

Proof. $(\Rightarrow)$ Assume that $R$ reaches $t$ starting from $s$ attached to some context. Then there is $u \in C_{\Sigma}$ such that $u[s] \rightarrow_{R}^{*} t$. If $u[s]=t$, then (i) holds. Otherwise, $u[s] \rightarrow_{R}^{+} t$. Hence there are $v_{1}, v_{2}, z \in C_{\Sigma}$ and a rule $l \rightarrow r$ in $R$ such that $u[s]=v_{1}[z[s]] \rightarrow_{R}^{*} v_{1}[l] \rightarrow_{R} v_{1}[r] \rightarrow_{R}^{*} v_{2}[r]=t$, where

(a) $u=v_{1}[z]$,

(b) $z[s] \rightarrow_{R}^{*} l$,

(c) $l \rightarrow r \in R$,

(d) $v_{1} \rightarrow_{R}^{*} v_{2}$ over the ranked alphabet $\Sigma \cup \diamond$.

Hence $t=v_{2}[r], v_{2} \in C_{\Sigma}, r \in \operatorname{rhs}(R)$. By (b), $s \in \operatorname{sub}(l)$ or $s \in \operatorname{sub}\left(l_{1}\right)$ for some $l_{1} \in L H S(R)$. Recall that $s \in \operatorname{IRR}(R)$. Hence $s \in(\operatorname{sub}(R)-l h s(R))$.

$(\Leftarrow)$ If (i) holds, then $R$ reaches $t$ starting from $s$ attached to some context.

Assume that (ii) holds. Then there is $z \in C_{\Sigma}$ such that $z[s] \rightarrow_{R}^{*} r$. Consequently $(u[z])[s]=u[z[s]] \rightarrow_{R}^{*} u[r]=t$. Hence $R$ reaches $t$ starting from $s$ attached to some context.

Lemma 1 and Lemma 2 imply the following result.

Proposition 5. For any $s, t \in I R R(R)$, we can decide whether $R$ reaches $t$ starting from $s$ attached to some context. 


\section{Two trivial semi-decision procedures}

We present the well known trivial semi-decision procedure PRO1 for the ground word problem of variable preserving TESs. We give examples when PRO1 is efficient. Then we present the trivial semi-decision procedure PRO2 for the ground word problem of TESs. Note that PRO2 is a straightforward generalization of PRO1.

Procedure PRO1 Input: A variable preserving TES $E$ over the ranked alphabet $\Sigma$ and ground terms $p, q \in T_{\Sigma}$.

Output: 'yes' if $p \leftrightarrow{ }_{E}^{*} q$, 'no' or undefined otherwise.

Let $U_{0}=\{p\}, V_{0}=\{q\}, i=0$.

repeat

$i:=i+1$;

$U_{i}:=U_{i-1} \cup\left\{s \mid\right.$ there is $u \in U_{i-1}$ such that $\left.u \leftrightarrow_{E} s\right\}$;

$V_{i}:=V_{i-1} \cup\left\{s \mid\right.$ there is $u \in V_{i-1}$ such that $\left.u \leftrightarrow_{E} s\right\}$;

until $\left(U_{i}=U_{i-1}\right.$ or $\left.V_{i}=V_{i-1}\right)$ or $U_{i} \cap V_{i}$ is not empty;

if $U_{i} \cap V_{i}$ is not empty

then begin output 'yes'; halt end;

output 'no';

halt

For any variable preserving TES $E$ and ground term $u$, the set $\left\{s \mid u \leftrightarrow_{E} s\right\}$ is finite and then effectively computable. Thus for every $i \geq 0, U_{i}$ and $V_{i}$, are finite and can be computed effectively. Hence the above procedure can be implemented. Clearly, PRO1 outputs 'yes' and halts if and only if $p \leftrightarrow \leftrightarrow_{E}^{*} q$. If PRO1 outputs 'no' and halts, then $(p, q) \notin \leftrightarrow_{E}^{*}$.

We adopt the following example of Lynch [13].

Example 2. Let $\Sigma=\Sigma_{0} \cup \Sigma_{1}, \Sigma_{0}=\{\$, \#\}, \Sigma_{1}=\{f, g\}$. Consider the TES $E=\{f f x \approx g f x\}$. We raise the problem whether $\$ \leftrightarrow_{E}^{*} \#$. On the one hand, the basic Knuth-Bendix completion procedure runs forever on this example [13]. On the other hand, the goal-directed completion procedure outputs 'no' and halts [13]. It is still open whether the goal-directed completion procedure halts on the TES $E$ and any goal [13].

Observe that for each $u \in T_{\Sigma}$, the set $\left\{s \mid u \leftrightarrow_{E}^{*} s\right\}$ is finite. Hence for any $p, q \in T_{\Sigma}$, PRO1 outputs the correct answer and halts. For this example, PRO1 is more efficient than the basic Knuth-Bendix completion procedure, and is at least as efficient as the goal-directed completion procedure [13, 14].

Example 3. Let $\Sigma=\Sigma_{0} \cup \Sigma_{2}, \Sigma_{0}=\{\star, \$, \#\}$, and $\Sigma_{2}=\{f\}$. We define the terms $\operatorname{comb}_{i} \in T_{\Sigma}\left(X_{i}\right), i \geq 1$, as follows. Let $\operatorname{comb}_{1}=f\left(x_{1}, \star\right), \operatorname{comb}_{i+1}=$ $f\left(x_{1}, \operatorname{comb}_{i}\left[x_{2}, \ldots, x_{i+1}\right]\right)$ for $i \geq 1$. For example, $\mathrm{comb}_{3}=f\left(x_{1}, f\left(x_{2}, f\left(x_{3}, \star\right)\right)\right)$. Let $n \geq 1, p=\operatorname{comb}_{2 n}[\#, \ldots, \#]$, and $q=\operatorname{comb}_{2 n}[\$, \ldots, \$]$. We run procedure PRO1 on the TES $E=\{\# \approx \$\}$ and the ground terms $p$ and $q$. Then

$$
\operatorname{card}\left(U_{i}\right)=\operatorname{card}\left(V_{i}\right)=\left(\begin{array}{c}
2 n \\
i
\end{array}\right)+\left(\begin{array}{c}
2 n \\
i-1
\end{array}\right)+\cdots+\left(\begin{array}{c}
2 n \\
1
\end{array}\right) \text { for } i=1, \ldots n,
$$


$U_{i} \cap V_{i}=\emptyset$ for $i=0,1, \ldots n-1$, and $\mathrm{comb}_{2 n}[\#, \ldots, \#, \$, \ldots, \$] \in U_{n} \cap V_{n}$.

Hence in the $n$th step, PRO1 outputs 'yes' and halts.

Example 4. We present Ceitin's [3,11] semi-Thue system as a TES. Let $\Sigma=$ $\Sigma_{0} \cup \Sigma_{1}, \Sigma_{0}=\{\$\}$, and $\Sigma_{1}=\{a, b, c, d, e\} . E$ consists of the equations

$a c x_{1} \approx c a x_{1}, a d x_{1} \approx d a x_{1}, b c x_{1} \approx c b x_{1}, b d x_{1} \approx d b x_{1}$, $e c a x_{1} \approx c e x_{1}, e d b x_{1} \approx d e x_{1}$

cdcax $_{1} \approx$ cdcaex $_{1}$, caaax $_{1} \approx a_{a a x}$, daaax $_{1} \approx a_{a a a x}$.

Proposition 6. $[3,11]$ It is undecidable for an arbitrary given ground term $t \in T_{\Sigma}$ whether $t \leftrightarrow \leftrightarrow_{E}^{*} a^{3} \$$.

We run procedure PRO1 on the TES $E$ and the ground terms $p=a^{3} \$$ and $q=e d b \$$. We compute as follows.

$U_{0}=\{p\}, V_{0}=\{q\}$,

$U_{1}=\left\{a^{3} \$, c a^{3} \$, d a^{3} \$\right\}, V_{1}=\{e d b \$, e b d \$, d e \$\}$,

$U_{2}=\left\{a^{3} \$, c a^{3} \$, d a^{3} \$, c c a^{3} \$, c d a^{3} \$, d c a^{3} \$, d d a^{3} \$, a c a a \$, a d a a \$\right\}, V_{2}=V_{1}$.

Now procedure $P R O 1$ outputs 'no' and halts.

Let $n \geq 1, p=(b d)^{2 n} \$$, and $q=(d b)^{2 n} \$$. We apply procedure PRO1 to TES $E$ and ground terms $p$ and $q$. We compute as follows.

$U_{0}=\{p\}, V_{0}=\{q\}$,

$U_{1}=\left\{p, d b(b d)^{2 n-1} \$, \ldots,(b d)^{2 n-1} d b \$\right\}$,

$V_{1}=\left\{q, b d(d b)^{2 n-1} \$, \ldots,(d b)^{2 n-1} b d \$,\right\}$,

$U_{2}=U_{1} \cup\left\{d b d b(b d)^{2 n-2} \$, d b b d d b(b d)^{2 n-3} \$, \ldots,(b d)^{2 n-2} d b d b \$\right\}$,

$V_{2}=V_{1} \cup\left\{b d b d(d b)^{2 n-2} \$, b d d b b d(d b)^{2 n-3} \$, \ldots,(d b)^{2 n-2} b d b d \$\right\}$,

....

Observe that $U_{i} \cap V_{i}=\emptyset$ for $i=0,1, \ldots, n-1$. Clearly, $(b d)^{n}(d b)^{n} \$ \in U_{n} \cap V_{n}$. After computing $U_{n}$ and $V_{n}$, procedure PRO1 outputs 'yes' and halts.

Example 5. We continue Example 4. Let $p \in T_{\Sigma}$ be arbitrary such that symbols $a$ or $c$ appear in $p$. Let $q \in T_{\Sigma}$ such that $a, c$ do not appear in $q$. That is, only the constant $\$$ and the symbols $b, d$, or $e$ appear in $q$.

Observe that the left-hand side and the right-hand side of the fourth and sixth rules do not contain $a$ or $c$. Both sides of all other rules contain $a$ or $c$. Hence for any reduction sequence

$p \rightarrow_{R} p_{1} \rightarrow_{R} p_{2} \rightarrow_{\ldots} \rightarrow_{R} p_{n}, n \geq 1$, for any $1 \leq i \leq n$, the term $p_{i}$ contains the constant $\$$ and at least one $a$ or $c$. Furthermore, along any reduction sequence $q \rightarrow_{R} q_{1} \rightarrow_{R} q_{2} \rightarrow_{\ldots} \rightarrow_{R} q_{n}, n \geq 1$, we only use the fourth and sixth equations. Consequently, the set $\left\{v \in T_{\Sigma} \mid q \leftrightarrow_{E}^{*} v\right\}$ is finite. Furthermore neither $a$ nor $c$ appears in any element of the set $\left\{v \in T_{\Sigma} \mid q \leftrightarrow \leftrightarrow_{E}^{*} v\right\}$. Thus

$$
(p, q) \notin \stackrel{\stackrel{*}{\leftrightarrow}}{E},
$$

and $U_{i} \cap V_{i}=\emptyset$ for $i \geq 0$. Thus procedure PRO1 outputs 'no' and halts on the input $E, p, q$. 
Example 6. Let $\Sigma=\Sigma_{0} \cup \Sigma_{1}, \Sigma_{0}=\{a\}$, and $\Sigma_{1}=\{f\}$. TES $E$ consists of the equation $f f x \approx x$. We run procedure PRO1 on TES $E$ and ground terms $p=a$ and $q=f a$. We compute as follows.

$U_{0}=\{a\}, V_{0}=\{f a\}$,

$U_{1}=\{a, f f a\}, V_{1}=\left\{f a, f^{3} a\right\}$,

$U_{2}=\left\{a, f f a, f^{4} a\right\}, V_{2}=\left\{f a, f^{3} a, f^{5} a\right\}, \ldots$

$U_{0} \subset U_{1} \subset U_{2} \subset \cdots$,

$V_{0} \subset V_{1} \subset V_{2} \subset \cdots$, and

$U_{i} \cap V_{i}=\emptyset$ for $i \geq 0$.

Hence procedure PRO1 does not halt.

To present the semi-decision procedure PRO2, we define the sets $U_{i} \subseteq T_{\Sigma}$, $i \geq 0$, by recursion. Let $U_{0}=\{p\}$. Let $i \geq 1$. We put all elements of $U_{i-1}$ in $U_{i}$. Moreover, we put in $U_{i}$ all $s \in T_{\Sigma}$ such that

- $l^{\prime} \approx r^{\prime}$ is a ground instance of some equation $l \approx r$ in $E \cup E^{-1}$ obtained by substituting arbitrary ground terms of height less than or equal to $i-1$ for all variables that do not appear in $l$,

- $v \in C_{\Sigma}$,

- $v\left[l^{\prime}\right] \in U_{i-1}$ and $s=v\left[r^{\prime}\right]$.

We define $V_{i} \subseteq T_{\Sigma}, i \geq 0$, symmetrically to $U_{i}, i \geq 0$. Clearly for every $i \geq 0, U_{i}$ and $V_{i}$ are finite and can be computed effectively. Note that there may be an $i \geq 1$ such that $U_{i}=U_{i+1}$ and $U_{i+1} \subset U_{i+2}$.

Example 7. Let $\Sigma=\Sigma_{0} \cup \Sigma_{1}, \Sigma_{0}=\{0,1\}$, and $\Sigma_{2}=\{f\}$. Let TES $E$ consist of the equations

$$
f\left(x_{1}, x_{1}\right) \approx 0, f\left(0, x_{1}\right) \approx x_{1} .
$$

Let $p=f(1,0)$ and $q=f(1, f(1,1))$. Then

$U_{0}=\{f(1,0)\}, V_{0}=\{f(1, f(1,1))\}$,

$U_{1}=\{f(1,0), f(f(0,1), 0), f(1, f(0,0)), f(1, f(1,1))\}$,

$V_{1}=\{f(1, f(1,1)), f(f(0,1), f(1,1)), f(1,0), f(1, f(f(0,1), 1))$,

$f(1, f(1, f(0,1)))\}$.

Procedure PRO2 Input: A TES $E$ over the ranked alphabet $\Sigma$ and ground terms $p, q \in T_{\Sigma}$.

Output: 'yes' if $p \leftrightarrow_{E}^{*} q$, undefined otherwise.

$1 \quad i:=i+1$;

compute $U_{i}$ and $V_{i}$;

if $U_{i} \cap V_{i}$ is not empty then begin output 'yes'; halt end;

goto 1

PRO2 outputs 'yes' and halts if and only if $p \leftrightarrow_{E}^{*} q$.

Example 8. We continue Example 7. We run procedure PRO2 on TES E and ground terms $p, q$. We compute as follows. We compute $U_{0}$ and $V_{0}$. We observe that $U_{0} \cap V_{0}$ is empty. Then we compute $U_{1}$ and $V_{1}$. We observe that $U_{1} \cap V_{1}$ is not empty. Procedure PRO2 outputs 'yes' and halts. 


\section{Semi-decision procedure for the ground word problem of variable preserving TESs}

We present the semi-decision procedure $P R O 3$ for the ground word problem of variable preserving TESs, and show its correctness. PRO3 is an improvement of PRO1. The starting idea is the following. For each $i \geq 1$, we construct the GTES $P_{i}$ using those instances of equations in $E \cup E^{-1}$ which are applied to compute the set $U_{i}$. We improve this construction by defining $P_{i}, i \geq 2$, as the set of all instances of equations in $E \cup E^{-1}$ which can be applied to elements of $\left\{s \in T_{\Sigma} \mid\right.$ $\left.p \leftrightarrow{ }_{P_{i-1}}^{*} s\right\}$ rather than to the elements of $U_{i-1}$. Furthermore, we define the GTES $Q_{i}$ symmetrically. We give examples when procedure $P R O 3$ is more efficient than procedure PRO1.

Let $E$ be a variable preserving TES over $\Sigma$, and let $p, q \in T_{\Sigma}$. We define the GTESs $P_{i}$ and the reduced GTRSs $R_{i}, i \geq 1$, over $\Sigma$ as follows.

For each equation $l \approx r$ of $E \cup E^{-1}$ with $l, r \in T_{\Sigma}\left(X_{m}\right), m \geq 0$, and for any $u \in C_{\Sigma}, u_{1}, \ldots, u_{m} \in T_{\Sigma}$, if $p=u\left[l\left[u_{1}, \ldots, u_{m}\right]\right]$ then we put the equation $l\left[u_{1}, \ldots, u_{m}\right] \approx r\left[u_{1}, \ldots, u_{m}\right]$ in $P_{1}$. Applying Snyder's algorithm we compute a reduced GTRS $R_{1}$ equivalent to the GTES $P_{1}$, see Proposition 3.

Let $i \geq 1$. (a) We put each element of $R_{i}$ into $P_{i+1}$.

(b) For each equation $l \approx r$ of $E \cup E^{-1}, l, r \in T_{\Sigma}\left(X_{m}\right), m \geq 0$, for any $u_{1}, \ldots, u_{m} \in\left(\operatorname{sub}\left(R_{i}\right)-\operatorname{lhs}\left(R_{i}\right)\right) \cup \operatorname{sub}\left(p \downarrow_{R_{i}}\right)$, if $R_{i}$ reaches $p \downarrow_{R_{i}}$ starting from $l\left[u_{1}, \ldots, u_{m}\right] \downarrow_{R_{i}}$ attached to some context, and $l\left[u_{1}, \ldots, u_{m}\right] \downarrow_{R_{i}} \neq r\left[u_{1}, \ldots, u_{m}\right] \downarrow_{R_{i}}$, then we put the equation $l\left[u_{1}, \ldots, u_{m}\right] \approx r\left[u_{1}, \ldots, u_{m}\right]$ in $P_{i+1}$.

If $P_{i+1}=R_{i}$, then let $R_{i+1}=R_{i}$. Otherwise, applying Snyder's algorithm, we compute a reduced GTRS $R_{i+1}$ equivalent to the GTES $P_{i+1}$.

When misunderstanding may arise, we denote $R_{i}$ as $R_{P_{i}}$. We define the GTESs $Q_{i}, i \geq 1$, symmetrically to the GTESs $P_{i}, i \geq 1$. Applying Snyder's algorithm, we compute a reduced GTRS $R_{P_{i} \cup Q_{i}}$ equivalent to the GTRS $R_{P_{i}} \cup R_{Q_{i}}$ for $i \geq 1$.

We illustrate our concepts and results by the following example.

Example 9. Let $\Sigma=\Sigma_{0} \cup \Sigma_{1} \cup \Sigma_{2}, \Sigma_{0}=\{\$, \#\}, \Sigma_{1}=\{e, f, g, h\}$, and $\Sigma_{2}=\{d\}$. Let the TES $E$ consist of the equations $\# \approx \$, \quad g \$ \approx h \$, \quad d\left(h x_{1}, h x_{1}\right) \approx h x_{1}, \quad e f h x_{1} \approx h x_{1}$.

Observe that $E$ is variable preserving. Let $p=e f g \#, q=d(h \#, h \#)$.

First we compute the GTES $P_{i}, i \geq 1$. GTES $P_{1}$ consists of the equation \# $\approx \$$. Let $\Theta$ stand for $\leftrightarrow_{P_{1}}^{*} \cap\left(\operatorname{sub}\left(P_{1}\right) \times \operatorname{sub}\left(P_{1}\right)\right)$. Then $\operatorname{sub}\left(P_{1}\right) / \Theta=\{\{\#, \$\}\}$ and $\{\$\}$ is a set of representatives for $\operatorname{sub}\left(P_{1}\right) / \leftrightarrow_{P_{1}}^{*}$. GTRS $R_{1}$ consists of the rule \# $\rightarrow \$$.

GTES $P_{2}$ consists of the equations \# $\approx \$, \quad g \$ \approx h \$$. Let $\Theta$ stand for $\leftrightarrow_{P_{2}}^{*} \cap\left(\operatorname{sub}\left(P_{2}\right) \times \operatorname{sub}\left(P_{2}\right)\right)$. Then $\operatorname{sub}\left(P_{2}\right) / \Theta=\{\{\#, \$\},\{g \#, g \$, h \#, h \$\}\}$ and $\{\$, h \$\}$ is a set of representatives for $\operatorname{sub}\left(P_{2}\right) / \leftrightarrow_{P_{2}}^{*}$. GTRS $R_{2}$ consists of the rules $\# \rightarrow \$, g \$ \rightarrow h \$$.

GTES $P_{3}$ consists of the equations

$\# \approx \$, \quad g \$ \approx h \$, \quad h \$ \approx d(h \$, h \$), \quad h \$ \approx e f h \$$.

Let $\Theta$ stand for $\leftrightarrow_{P_{3}}^{*} \cap\left(\operatorname{sub}\left(P_{3}\right) \times \operatorname{sub}\left(P_{3}\right)\right)$. Then 
$\operatorname{sub}\left(P_{3}\right) / \Theta=\{\{\#, \$\},\{g \#, g \$, h \#, h \$, d(h \$, h \$), e f h \$\},\{f h \$\}\}$

and $\{\$, h \$, f h \$\}$ is a set of representatives for $s u b\left(P_{3}\right) / \leftrightarrow_{P_{3}}^{*} \cdot R_{3}$ consists of the rules

$\# \rightarrow \$, \quad g \$ \rightarrow h \$, \quad d(h \$, h \$) \rightarrow h \$, \quad$ efh $\$ \rightarrow h \$$.

$P_{4}=R_{3}$ and $R_{4}=R_{3}$. Furthermore, $P_{i}=R_{3}$ and $R_{i}=R_{3}$ for $i \geq 4$.

Second, we compute the GTESs $Q_{i}, i \geq 1$. GTES $Q_{1}$ consists of the equations $\# \approx \$, d(h \#, h \#) \approx h \#$. GTRS $R_{Q_{1}}$ consists of the rules $\# \rightarrow \$, d(h \$, h \$) \rightarrow h \$$.

GTES $Q_{2}$ consists of the equations $\# \approx \$, d(h \$, h \$) \approx h \$$, ef $h \$ \approx h \$$.

GTRS $R_{Q_{2}}$ consists of the rules $\# \rightarrow \$, d(h \$, h \$) \rightarrow h \$$, ef $h \$ \rightarrow h \$$.

Observe that $R_{Q_{2}}=Q_{i}=R_{Q_{i}}$ for $i \geq 3$.

$R_{P_{1} \cup Q_{1}}=R_{P_{1}}, R_{P_{2} \cup Q_{2}}=R_{P_{2}} \cup R_{Q_{2}}$, and $R_{P_{3} \cup Q_{3}}=R_{P_{3}}$. Then

$p \downarrow_{R_{P_{1} \cup Q_{1}}}=$ efg $\$$, $q \downarrow_{R_{P_{1} \cup Q_{1}}}=h \$$,

$p \downarrow_{R_{P_{2}} \cup Q_{2}}=h \$, q \downarrow_{R_{P_{2}} \cup Q_{2}}=h \$$.

We get the following result by direct inspection of the definition of the GTES $P_{i}, i \geq 1$.

Lemma 3. (a) For each $i \geq 1, \leftrightarrow_{P_{i}}^{*}=\leftrightarrow_{R_{i}}^{*} \subseteq \leftrightarrow_{P_{i+1}}^{*} \subseteq \leftrightarrow_{E}^{*}$.

(b) If $R_{i}=P_{i+1}$ for some $i \geq 1$, then $R_{i}=P_{j}=R_{j}$ for $j \geq i+1$.

Lemma 4. For each $i \geq 1$, we can effectively construct the GTES $P_{i}$.

Proof. By induction on $i$.

Base Case: $i=1$. Clearly, we can construct $P_{1}$.

Induction Step: Let $i \geq 1$. Assume that we have constructed $P_{i}$. By Proposition 3 , we can construct $R_{i}$. Consider item (b) in the definition of $P_{i}$. By Proposition 5 , we can effectively decide whether $R_{i}$ reaches $p \downarrow_{R_{i}}$ starting from $l\left[u_{1} \ldots, u_{m}\right] \downarrow_{R_{i}}$ attached to some context. Hence we can construct $P_{i+1}$ as well.

We now present our semi-decision procedure.

Procedure PRO3 Input: A variable preserving TES $E$ over the ranked alphabet $\Sigma$ and ground terms $p, q \in T_{\Sigma}$.

Output: • 'yes' if $p \leftrightarrow_{E}^{*} q$,

- 'no' if $(p, q) \notin \leftrightarrow_{E}^{*}$ and the procedure halts,

- undefined if the procedure does not halt.

compute $P_{1}, R_{P_{1}}, Q_{1}, R_{Q_{1}}$, and $R_{P_{1} \cup Q_{1}}$;

if $p \downarrow_{R_{P_{1} \cup Q_{1}}}=q \downarrow_{R_{P_{1} \cup Q_{1}}}$, then begin output 'yes'; halt end;

$i:=1$;

1: $i:=i+1$;

compute $P_{i}, R_{P_{i}}, Q_{i}, R_{Q_{i}}$, and $R_{P_{i} \cup Q_{i}}$;

if $p \downarrow_{R_{P_{i} \cup Q_{i}}}=q \downarrow_{R_{P_{i} \cup Q_{i}}}$, then begin output 'yes'; halt end;

if $R_{P_{i-1}}=P_{i}$ or $R_{Q_{i-1}}=Q_{i}$,

then begin output 'no'; halt end;

goto 1 
Example 10. We continue Example 9. Note that $p \downarrow_{R_{P_{1} \cup Q_{1}}} \neq q \downarrow_{R_{P_{1} \cup Q_{1}}}$. Hence procedure $\mathrm{PRO} 3$ does not output anything and does not halt in the first step. Observe that $p \downarrow_{R_{P_{2}} \cup Q_{2}}=q \downarrow_{R_{P_{2}} \cup Q_{2}}$. Hence procedure PRO3 outputs 'yes' and halts in the second step.

Example 11. We continue Example 5. Let $n \geq 1$. We run procedure PRO3 on the TES $E$ and the ground terms $p=(b d)^{2 n} \$$, and $q=(d b)^{2 n} \$$. We compute as follows. GTES $P_{1}$ consists of the equation $b d \$ \approx d b \$$. Let $\Theta$ stand for $\leftrightarrow_{P_{1}}^{*} \cap\left(\operatorname{sub}\left(P_{1}\right) \times \operatorname{sub}\left(P_{1}\right)\right)$. Then $\operatorname{sub}\left(P_{1}\right) / \Theta=\{\{b \$\},\{d \$\},\{b d \$\}\}$ and $\{b d \$\}$ is a set of representatives for $\operatorname{sub}\left(P_{1}\right) / \leftrightarrow_{P_{1}}^{*}$. GTRS $R_{P_{1}}$ consists of the rule $b d \$ \rightarrow d b \$$.

Symmetrically, GTES $Q_{1}$ consists of the equation $d b \$ \approx b d \$$. GTRS $R_{Q_{1}}$ consists of the rule $d b \$ \rightarrow b d \$$. It is not hard to see, that GTRS $R_{P_{1} \cup Q_{1}}$ is equal to GTRS $R_{P_{1}}$. Observe that $p \downarrow_{R_{P_{1} \cup Q_{1}}}=q \downarrow_{R_{P_{1} \cup Q_{1}}}$, Hence procedure PRO3 outputs 'yes' and halts in the first step.

We run procedure PRO3 on the TES $E$ and the ground terms $p=a a a \$$ and $q=b e d b \$$. By our arguments in Example 5,

$$
p \downarrow_{R_{P_{i}} \cup Q_{i}} \neq q \downarrow_{R_{P_{i} \cup Q_{i}}} \quad \text { for } i \geq 1 \text {. }
$$

Furthermore, $\mathrm{PRO} 3$ computes as follows.

$$
\begin{aligned}
& R_{Q_{1}}=\{d b \$ \rightarrow b d \$, e d b \$ \rightarrow d e \$\}, \\
& R_{Q_{2}}=\{d b \$ \rightarrow b d \$, e d b \$ \rightarrow d e \$, b d d e \$ \rightarrow d b d e \$\}, \text { and } \\
& R_{Q_{2}}=R_{Q_{n+2}} \text { for } n \geq 1 .
\end{aligned}
$$

Consequently, Procedure PRO3 outputs 'no' and then halts. Generalizing our arguments, we can show the following.

Statement 1. Let $p \in T_{\Sigma}$ be arbitrary such that symbols a or $c$ appear in $p$. Let $q \in T_{\Sigma}$ such that a, $c$ do not appear in $q$. Then procedure PRO3 outputs 'no' and halts on the input $E, p, q$.

By Propositon 6, for an arbitrary ground term $q^{\prime} \in T_{\Sigma}$, the goal-directed completion procedure [13] may fail or may not halt on the TES $E$ and the goal $\left(a a a \$, q^{\prime}\right)$. The following problem is open. For each goal $(a a a \$, q)$ such that $q \in T_{\Sigma}$, and $a, c$ do not appear in $q$, is it true that the the goal-directed completion procedure does not fail and halts on the TES $E$ and the goal $(a a a \$, q)$.

It is open whether the goal-directed completion procedure does not fail and halts on the TES $E$ and any goal $(a a a \$, q)$ such that $q \in T_{\Sigma}, a, c$ do not appear in $q$.

We now show the correctness of Procedure PRO3.

Lemma 5. For any $i, n$ with $1 \leq n \leq i$, and any $t_{1}, \ldots, t_{n} \in T_{\Sigma}$, if $p \leftrightarrow_{E} t_{1} \leftrightarrow_{E} t_{2} \leftrightarrow \leftrightarrow_{E} \cdots \leftrightarrow_{E} t_{n}$, then $p \leftrightarrow_{P_{i}}^{*} t_{1} \leftrightarrow_{P_{i}}^{*} t_{2} \leftrightarrow_{P_{i}}^{*} \cdots \leftrightarrow_{P_{i}}^{*} t_{n}$.

Proof. We proceed by induction on $i$.

Base Case: $i=1$. Then $n=1$. By the definition of $P_{1}$, we have $p \leftrightarrow_{P_{1}} t_{1}$. 
Induction Step: Let $i \geq 1$, and assume that the statement holds for $1,2, \ldots, i$. We now show that the statement holds for $i+1$. To this end, assume that

$$
p \underset{E}{\leftrightarrow} t_{1} \underset{E}{\overleftrightarrow{㇒}} t_{2} \underset{E}{\leftrightarrow} \cdots \underset{E}{\leftrightarrow} t_{n} \text { for some } 0 \leq n \leq i+1
$$

By the induction hypothesis,

$$
p \underset{P_{i}}{\stackrel{*}{\rightarrow}} t_{1} \underset{P_{i}}{\stackrel{*}{\leftrightarrow}} t_{2} \underset{P_{i}}{\stackrel{*}{\rightarrow}} \cdots \stackrel{\stackrel{*}{\leftrightarrow}}{\stackrel{\leftrightarrow}{P_{i}}} t_{n-1} .
$$

Hence

$$
t_{n-1} \underset{R_{i}}{\stackrel{*}{\rightarrow}} p \downarrow_{R_{i}}
$$

By (6), there is an equation $l \approx r$ in $E \cup E^{-1}$ with $l, r \in T_{\Sigma}\left(X_{m}\right), m \geq 0$ and there are $u \in C_{\Sigma}, u_{1}, \ldots, u_{m} \in T_{\Sigma}$ such that

$$
t_{n-1}=u\left[l\left[u_{1}, \ldots, u_{m}\right]\right] \text { and } t_{n}=u\left[r\left[u_{1}, \ldots, u_{m}\right]\right] .
$$

As $R_{i}$ is convergent, by (8) and (9), $u\left[l\left[u_{1}, \ldots, u_{m}\right] \downarrow_{R_{i}}\right] \rightarrow_{R_{i}}^{*} p \downarrow_{R_{i}}$. That is, $R_{i}$ reaches $p \downarrow_{R_{i}}$ starting from $l\left[u_{1}, \ldots, u_{m}\right] \downarrow_{R_{i}}$ attached to some context. By the definition of $P_{i+1}$,

$$
l\left[u_{1}, \ldots, u_{m}\right] \approx r\left[u_{1}, \ldots, u_{m}\right] \text { is in } \underset{P_{i}}{\stackrel{*}{\leftrightarrow}} \text { or } P_{i+1} .
$$

By Lemma 3, (7), (9), and (10),

$$
p \underset{P_{i+1}}{\stackrel{*}{\leftrightarrow}} t_{1} \underset{P_{i+1}}{\stackrel{*}{\leftrightarrow}} t_{2} \underset{P_{i+1}}{\stackrel{*}{\leftrightarrow}} \cdots \underset{P_{i+1}}{\stackrel{*}{\leftrightarrow}} t_{n-1} \underset{P_{i+1}}{\stackrel{*}{\leftrightarrow}} t_{n}
$$

By Lemma 3 and Lemma 5 we have the following result.

Lemma 6. Assume that $R_{i}=P_{i+1}$ for some $i \geq 1$. Then $p \leftrightarrow_{P_{i+1}}^{*} q$ if and only if $p \leftrightarrow{ }_{E}^{*} q$.

Theorem 1. If $p \leftrightarrow_{E}^{*} q$, then procedure PRO3 outputs 'yes' and halts.

Proof. Assume that $p=t_{1} \leftrightarrow_{E} t_{2} \leftrightarrow_{E} \cdots \leftrightarrow_{E} t_{n}=q$ for some $n \geq 1$ and $t_{1}, \ldots, t_{n} \in T_{\Sigma}$. By Lemma $5, p \leftrightarrow_{P_{n}}^{*} q$. Let $k$ be the least integer such that $p \leftrightarrow{ }_{P_{k} \cup Q_{k}}^{*} q$.

First assume that $k=1$. Then $p \leftrightarrow_{P_{1} \cup Q_{1}}^{*} q$. Hence $p \downarrow_{R_{P_{1} \cup Q_{1}}}=q \downarrow_{R_{P_{1} \cup Q_{1}}}$. Consequently, procedure PRO3 outputs 'yes' and halts in the first step.

Second assume that $k \geq 2$. Then by the definition of $k,(p, q) \notin \leftrightarrow_{P_{i} \cup Q_{i}}^{*}$ for $2 \leq i \leq k-1$. Then by Lemma $6, R_{P_{i-1}} \subset P_{i}$ and $R_{Q_{i-1}} \subset Q_{i}$ for $2 \leq i \leq k-1$. Hence procedure $P R O 3$ does not halt in the first $k-1$ steps. By the definition of the integer $k$, in the $k$ th step procedure PRO3 outputs 'yes' and halts. 
Theorem 2. If procedure PRO3 outputs 'yes' and halts, then $p \leftrightarrow_{E}^{*} q$. If procedure PRO3 outputs 'no' and halts, then $(p, q) \notin \leftrightarrow_{E}^{*}$.

Proof. Assume that procedure PRO3 outputs 'yes' and halts in the $k$ th step. Then $p \leftrightarrow{ }_{P_{k}}^{*} \cup Q_{k} q$. By Lemma $3, p \leftrightarrow_{E}^{*} q$.

Assume that procedure PRO3 outputs 'no' and halts in the $k$ th step. Then

(a) $(p, q) \notin \leftrightarrow_{P_{k} \cup Q_{k}}^{*}$ and

(b) $P_{k}=R_{P_{k-1}}$ or $Q_{k}=R_{Q_{k-1}}$.

We now distinguish two cases.

Case 1: $P_{k}=R_{P_{k-1}}$. By (a) and by Lemma $6,(p, q) \notin \leftrightarrow_{E}^{*}$.

Case 2: $Q_{k}=R_{Q_{k-1}}$. This case is symmetric to Case 2 .

Theorems 1 and 2 imply the following.

Theorem 3. If $p \leftrightarrow_{E}^{*} q$, then procedure PRO3 outputs 'yes' and halts. Otherwise, either PRO3 outputs 'no' and halts, or PRO3 does not halt.

Example 12. We continue Example 3. We now run procedure PRO3 on the TES $E$ and the ground terms $p, q$. Then $P_{1}=Q_{1}=\{\# \approx \$\}, R_{P_{1}}=R_{Q_{1}}=P_{1}$, and $R_{P_{1} \cup Q_{1}}=P_{1}$. Observe that $p \downarrow_{R_{P_{1} \cup Q_{1}}}=q \downarrow_{R_{P_{1} \cup Q_{1}}}$. Hence procedure PRO3 outputs 'yes' and halts in the first step. By Proposition 2 , we compute $p \downarrow_{R_{P_{1} \cup Q_{1}}}$ and $q \downarrow_{R_{P_{1} \cup Q_{1}}}$ in linear time. We apply the rules of $R_{P_{1} \cup Q_{1}} n$ times. For this example, PRO3 is faster than PRO1.

Example 13. We continue Example 6. We now run procedure PRO3 on the TES $E$ and the ground terms $p$ and $q$. Then $\{a \approx f f a\}=P_{1}=R_{P_{1}}=P_{1+i}=R_{P_{1+i}}$ for $i \geq 1$. Furthermore, $Q_{1}=\{a \approx f f a, f a \approx f f f a\}, R_{Q_{1}}=P_{1}=Q_{2}=R_{Q_{2}}=$ $Q_{1+i}=R_{Q_{1+i}}$ for $i \geq 1$.

Observe that $p \downarrow_{R_{P_{2} \cup Q_{2}}} \neq q \downarrow_{R_{P_{2} \cup Q_{2}}}$. Hence procedure PRO3 outputs 'no' and halts in the second step.

It should be clear that for all ground terms $p$ and $q, P R O 3$ halts. It outputs 'yes' if $p \leftrightarrow_{E}^{*} q$. Otherwise it outputs 'no'.

Example 14. We now continue Example 2. We apply procedure PRO3 to the TES $E=\{f f x \approx g f x\}$ and any terms $p, q \in T_{\Sigma}$. Observe that height $(f f x)=$ $2=\operatorname{height}(g f x)$.

Statement 2. For each $i \geq 0$, and for each pair of terms, $s, t \in T_{\Sigma}(X)$, if $(s, t) \in$ $P_{i}$, then height $(s)=$ height $(t) \leq$ height $(p)$.

Proof. We proceed by induction on $n$.

Base Case: $i=1$. By the definition of $P_{1}$, for each equation $s \approx t$ in $P_{1}$, height $(s)=$ height $(t) \leq$ height $(p)$. Hence our statement holds.

Induction Step: Let $n \geq 1$, and assume that the satement holds for $1,2, \ldots, n$. We now show that the satement holds for $n+1$. Consider an equation

$l\left[u_{1}, \ldots, u_{m}\right] \approx r\left[u_{1}, \ldots, u_{m}\right]$ in $P_{i+1}$. Then there exist

- an equation $l \approx r$ of $E \cup E^{-1}$, where $l, r \in T_{\Sigma}\left(X_{m}\right), m \geq 0$. 
- $u_{1}, \ldots, u_{m} \in\left(\operatorname{sub}\left(R_{i}\right)-l h s\left(R_{i}\right)\right) \cup \operatorname{sub}\left(p \downarrow_{R_{i}}\right)$.

such that $R_{i}$ reaches $p \downarrow_{R_{i}}$ starting from $l\left[u_{1}, \ldots, u_{m}\right] \downarrow_{R_{i}}$ attached to some context, and that

$l\left[u_{1}, \ldots, u_{m}\right] \downarrow_{R_{i}} \neq r\left[u_{1}, \ldots, u_{m}\right] \downarrow_{R_{i}}$.

Consequently, there is a $u \in C_{\Sigma}$ such that $u\left[l\left[u_{1}, \ldots, u_{m}\right]\right] \rightarrow_{R_{i}}^{*} p$. By (a) in Lemma 3 and the induction hypothesis, height $\left(u\left[l\left[u_{1}, \ldots, u_{m}\right]\right]\right)=h e i g h t(p)$. Thus $h e i g h t\left(l\left[u_{1}, \ldots, u_{m}\right]\right) \leq \operatorname{height}(p)$. By (a) in Lemma 3 and the induction hypothesis, height $(l)=$ height $(r)$. Hence height $\left(l\left[u_{1}, \ldots, u_{m}\right]\right)=\operatorname{height}\left(r\left[u_{1}, \ldots, u_{m}\right]\right)$.

Observe that the set $\left\{(s, t) \in T_{\Sigma} \times T_{\Sigma} \mid\right.$ height $(s)=$ height $(t) \leq$ height $\left.(p)\right\}$ is finite. By Lemma 3 and Statement 2, procedure PRO3 halts on $E$ and any terms $p, q \in T_{\Sigma}$ in finitely many steps.

The following result can be shown by generalizing the proof appearing in Example 14

Theorem 4. Let $E$ be a variable preserving TES such that

- for any equation $s \approx t$ in $E$, height $(s)=$ height $(t)$, or

- for any equation $s \approx t$ in $E$, size $(s)=$ size $(t)$ and each variable appears the same times in $s$ and $t$.

Let $p, q \in T_{\Sigma}$ be arbitrary. Then procedure PRO3 halts on $E$ and terms $p, q$.

\section{Semi-decision procedure for the ground word problem of TESs}

We present the semi-decision procedure $\mathrm{PRO}_{4}$ for the ground word problem of TESs, and show its correctness. We obtain it generalizing PRO3 taking into account PRO2. The starting point to the definition of the GTESs $P_{i}, i \geq 1$, is the same as in Section 5. We define $P_{1}$ as the set of all instances $l^{\prime} \rightarrow r^{\prime}$ of equations $l \approx r$ in $E \cup E^{-1}$ which can be applied to $p$. We define $P_{i+1}, i \geq 1$, as the set of all instances $l^{\prime} \rightarrow r^{\prime}$ of equations $l \approx r$ in $E \cup E^{-1}$ which can be applied to elements of $\left\{s \in T_{\Sigma} \mid p \leftrightarrow P_{P_{i}}^{*} s\right\}$. The question is what should we substitute for those variables in the right-hand side $r$ that do not appear in the left-hand side $l$. We now give a simplified answer to this question. Applying Snyder's algorithm we compute a reduced GTRS $R_{i}$ equivalent to the GTES $P_{i}$. When constructing the instance $l^{\prime} \rightarrow r^{\prime}$ of $l \approx r$, we substitute any term in $\left(s u b\left(R_{i}\right)-l h s\left(R_{i}\right)\right) \cup \operatorname{sub}\left(p \downarrow_{R_{i}}\right)$ or the $R_{i}$ normal form of any ground term of height less than or equal to $i$ for each variable in the right-hand side $r$ that does not appear on the left-hand side $l$. Furthermore, we define the GTESs $Q_{i}, i \geq 1$, symmetrically.

Let $E$ be a TES over $\Sigma$, and let $p, q \in T_{\Sigma}$. We now define the GTESs $P_{i}$ and the reduced GTRSs $R_{i}, i \geq 1$, over $\Sigma$.

Let $N O R M_{0}=\Sigma_{0} \cup \operatorname{sub}(p)$. For each equation $l \approx r$ of $E \cup E^{-1}$ with $l \in T_{\Sigma}\left(X_{k+m}\right), r \in T_{\Sigma}\left(X_{k} \cup X_{[k+m+1, k+m+\ell]}\right)$ for some $k, m, \ell \geq 0$, if $p=$ $u\left[l\left[u_{1}, \ldots, u_{k+m}\right]\right]$ for some $u \in C_{\Sigma}, u_{1}, \ldots, u_{k+m} \in T_{\Sigma}$, then for all 
$v_{k+m+1}, \ldots, v_{k+m+\ell} \in N O R M_{0}$, we put the equation

$$
l\left[u_{1}, \ldots, u_{k+m}\right] \approx r\left[u_{1}, \ldots, u_{k}, v_{k+m+1}, \ldots, v_{k+m+\ell}\right]
$$

in $P_{1}$. Applying Snyder's algorithm we compute a reduced GTRS $R_{1}$ equivalent to the GTES $P_{1}$, see Proposition 3.

Let $i \geq 1$. Let

$\operatorname{NOR} M_{i}=\operatorname{sub}\left(p \downarrow_{R_{i}}\right) \cup\left(\operatorname{sub}\left(R_{i}\right)-\operatorname{lh} s\left(R_{i}\right)\right) \cup$

$\left\{t \downarrow_{R_{i}} \mid t \in N O R M_{i-1}\right.$ or $t=f\left(t_{1}, \ldots, t_{m}\right)$ for some $f \in \Sigma_{m}$ and $t_{1}, \ldots, t_{m} \in$ $\left.N O R M_{i-1}\right\}$.

(a) We put each rule of $R_{i}$ into $P_{i+1}$.

(b) For each equation $l \approx r$ of $E \cup E^{-1}$ with $l \in T_{\Sigma}\left(X_{k+m}\right), r \in T_{\Sigma}\left(X_{k} \cup\right.$ $\left.X_{[k+m+1, k+m+\ell]}\right)$ for some $k, m, \ell \geq 0$, for any $u_{1}, \ldots, u_{k+m} \in\left(\operatorname{sub}\left(R_{i}\right)-\operatorname{lh} s\left(R_{i}\right)\right) \cup$ $\operatorname{sub}\left(p \downarrow_{R_{i}}\right)$ and $v_{k+m+1}, \ldots, v_{k+m+\ell} \in N O R M_{i}$, if $R_{i}$ reaches $p \downarrow_{R_{i}}$ starting from $l\left[u_{1}, \ldots, u_{k+m}\right] \downarrow_{R_{i}}$ attached to some context, and

$l\left[u_{1}, \ldots, u_{k+m}\right] \downarrow_{R_{i}} \neq r\left[u_{1}, \ldots, u_{m}, v_{k+m+1}, \ldots, v_{k+m+\ell}\right] \downarrow_{R_{i}}$,

then we put the equation

$$
l\left[u_{1}, \ldots, u_{k+m}\right] \approx r\left[u_{1}, \ldots, u_{m}, v_{k+m+1}, \ldots, v_{k+m+\ell}\right]
$$

in $P_{i+1}$.

If we do not put equations in $P_{i+1}$ in item (b), i.e. $P_{i+1}=R_{i}$, then let $R_{i+1}=$ $R_{i}$. Otherwise, applying Snyder's algorithm, we compute a reduced GTRS $R_{i+1}$ equivalent to the GTES $P_{i+1}$.

When misunderstanding may arise, we denote $R_{i}$ as $R_{P_{i}}$. We define the GTESs $Q_{i}, i \geq 1$, symmetrically to the GTESs $P_{i}, i \geq 1$. Applying Snyder's algorithm, we compute a reduced GTRS $R_{P_{i} \cup Q_{i}}$ equivalent to the GTRS $R_{P_{i}} \cup R_{Q_{i}}$ for $i \geq 1$.

By Proposition 1 GTRSs $R_{P_{i}}, R_{Q_{i}}$, and $R_{P_{i} \cup Q_{i}}$ are convergent.

We illustrate our concepts and results by two running examples, each of them is presented as a series of examples.

Example 15. We continue Example 7. Let $p=f(0,1)$ and $q=f(f(0,1), 1)$. Observe that for any $u, v \in T_{\Sigma}$, if $u \leftrightarrow_{E}^{*} v$, then the parity of the number of 1 's in $u$ equals to that in $v$. Hence

$$
(p, q) \notin \underset{E}{\stackrel{*}{\leftrightarrow}} .
$$

We now construct the GTESs $P_{1}, P_{2}$, and $P_{3}$. Then $N O R M_{0}=\{0,1, f(0,1)\}$. $P_{1}$ consists of the equations

$$
\begin{aligned}
& 0 \approx f(0,0), \quad 0 \approx f(1,1), \quad 0 \approx f(f(0,1), f(0,1)), \\
& 1 \approx f(0,1), \quad f(0,1) \approx 1, \quad f(0,1) \approx f(0, f(0,1)) .
\end{aligned}
$$

$R_{1}$ consists of the rules

$$
f(0,0) \rightarrow 0, \quad f(1,1) \rightarrow 0, \quad f(0,1) \rightarrow 1 .
$$

NORM $M_{1}=\{0,1, f(1,0)\} . P_{2}$ consists of the equations

$f(0,0) \approx 0, \quad f(1,1) \approx 0, \quad f(0,1) \approx 1, \quad 0 \approx f(f(1,0), f(1,0))$.

$R_{2}$ consists of the rules

$$
f(0,0) \rightarrow 0, \quad f(1,1) \rightarrow 0, \quad f(0,1) \rightarrow 1, \quad f(f(1,0), f(1,0)) \rightarrow 0 .
$$


$\mathrm{NORM}_{2}=\{0,1, f(1,0), f(0, f(1,0)), f(1, f(1,0)), f(f(1,0), 0), f(f(1,0), 1)\}$. $P_{3}$ consists of the equations

$f(0,0) \approx 0, \quad f(1,1) \approx 0, \quad f(0,1) \approx 1, \quad f(f(1,0), f(1,0)) \approx 0$,

$0 \approx f(f(0, f(1,0)), f(0, f(1,0)))$

$0 \approx f(f(1, f(1,0)), f(1, f(1,0)))$,

$0 \approx f(f(f(1,0), 0), f(f(1,0), 0))$,

$0 \approx f(f(f(1,0), 1), f(f(1,0), 1))$.

$R_{3}$ consists of the rules

$$
\begin{aligned}
& f(0,0) \rightarrow 0, \quad f(1,1) \rightarrow 0, \quad f(0,1) \rightarrow 1, \quad f(f(1,0), f(1,0)) \rightarrow 0, \\
& f(f(0, f(1,0)), f(0, f(1,0))) \rightarrow 0, \\
& f(f(1, f(1,0)), f(1, f(1,0))) \rightarrow 0, \\
& f(f(f(1,0), 0), f(f(1,0), 0)) \rightarrow 0, \\
& f(f(f(1,0), 1), f(f(1,0), 1)) \rightarrow 0 .
\end{aligned}
$$

Continuing in this manner we get that

$$
R_{P_{i}} \subset R_{P_{i+1}} \text { for } i \geq 1 \text {. }
$$

We now compute the GTESs $Q_{1}, Q_{2}$, and $Q_{3}$.

$N O R M_{0}=\{0,1, f(0,1), f(f(0,1), 1)\}$.

$Q_{1}$ consists of the equations

$$
\begin{aligned}
& 0 \approx f(0,0), \quad 0 \approx f(1,1), \quad 0 \approx f(f(0,1), f(0,1)), \\
& 0 \approx f(f(f(0,1), 1), f(f(0,1), 1)), \\
& 1 \approx f(0,1), \quad f(0,1) \approx f(0, f(0,1)), \quad f(f(0,1), 1) \approx f(0, f(f(0,1), 1)) .
\end{aligned}
$$

$R_{Q_{1}}$ consists of the rules

$$
f(0,0) \rightarrow 0, \quad f(1,1) \rightarrow 0, \quad f(0,1) \rightarrow 1 .
$$

$N O R M_{1}=\{0,1, f(1,0)\}$

$Q_{2}$ consists of the equations

$$
f(0,0) \approx 0, \quad f(1,1) \approx 0, \quad f(0,1) \approx 1, \quad 0 \approx f(f(1,0), f(1,0)) .
$$

$R_{Q_{2}}$ consists of the rules

$$
\begin{aligned}
& f(0,0) \rightarrow 0, \quad f(1,1) \rightarrow 0, \quad f(0,1) \rightarrow 1, \quad f(f(1,0), f(1,0)) \rightarrow 0 . \\
& \text { NORM }_{2}=\{0,1, f(1,0), f(0, f(1,0)), f(1, f(1,0)), f(f(1,0), 0), f(f(1,0), 1)\} .
\end{aligned}
$$

$Q_{3}$ consists of the equations

$$
\begin{aligned}
& f(0,0) \approx 0, \quad f(1,1) \approx 0, \quad f(0,1) \approx 1, \quad 0 \approx f(f(1,0), f(1,0)), \\
& 0 \approx f(f(0, f(1,0)), f(0, f(1,0))), \quad 0 \approx f(f(1, f(1,0)), f(1, f(1,0))), \\
& 0 \approx f(f(f(1,0), 0), f(f(1,0), 0)), \quad 0 \approx f(f(f(1,0), 1), f(f(1,0), 1)) . \\
& R_{Q_{3}} \text { consists of the rules } \\
& \quad f(0,0) \rightarrow 0, \quad f(1,1) \rightarrow 0, \quad f(0,1) \rightarrow 1, \quad f(f(1,0), f(1,0)) \rightarrow 0, \\
& \quad f(f(0, f(1,0)), f(0, f(1,0))) \rightarrow 0, \quad f(f(1, f(1,0)), f(1, f(1,0))) \rightarrow 0, \\
& f(f(f(1,0), 0), f(f(1,0), 0)) \rightarrow 0, \quad f(f(f(1,0), 1), f(f(1,0), 1)) \rightarrow 0 .
\end{aligned}
$$

Continuing in this manner we get that

$$
R_{Q_{i}} \subset R_{Q_{i+1}} \text { for } i \geq 1
$$

Let $R_{P_{1} \cup Q_{1}}=R_{P_{1}}, R_{P_{2} \cup Q_{2}}=R_{P_{2}}$, and $R_{P_{3} \cup Q_{3}}=R_{P_{3}} \cup R_{Q_{3}}$. 
Example 16. Let $\Sigma=\Sigma_{0} \cup \Sigma_{1}, \Sigma_{0}=\{0,1\}$, and $\Sigma_{1}=\{g, h\}$. Let TES $E$ consist of the equations

$g x_{1} \approx x_{1}, \quad h x_{1} \approx h x_{2}$.

Let $p=0$ and $q=1$.

We now construct the GTESs $P_{1}, P_{2}$, and $P_{3}$. Then $N O R M_{0}=\{0,1\} . P_{1}$ consists of the equation $0 \approx g 0$.

$R_{1}$ consists of the rule $g 0 \rightarrow 0$.

$N O R M_{1}=\{0,1, g 1, h 0, h 1\}$.

$P_{2}=R_{1}$ and $R_{2}=P_{2}$.

$N O R M_{2}=\{0,1, g 1, h 0, h 1, g g 1, h g 1, g h 0, h h 0, g h 1, h h 1\}$.

$P_{3}=R_{2}$ and $R_{3}=P_{3}$.

We now construct the GTESs $Q_{1}, Q_{2}$, and $Q_{3}$. Then $N O R M_{0}=\{0,1\}$. $Q_{1}$ consists of the equation $1 \approx g 1$.

$R_{Q_{1}}$ consists of the rule $g 1 \rightarrow 1$.

$N O R M_{1}=\{0,1, g 0, h 0, h 1\}$.

$Q_{2}=R_{Q_{1}}$ and $R_{Q_{2}}=Q_{2}$.

$\mathrm{NORM}_{2}=\{0,1, g 0, h 0, h 1, g g 0, h g 0, g h 0, h h 0, g h 1, h h 1\}$.

$Q_{3}=R_{Q_{2}}$ and $R_{Q_{3}}=Q_{3}$.

$R_{P_{1}} \cup R_{Q_{1}}=R_{P_{1} \cup Q_{1}}=R_{P_{2} \cup Q_{2}}=R_{P_{3} \cup Q_{3}}$.

We get the following result by direct inspection of the definition of the GTES $P_{i}$ and GTRS $R_{i}, i \geq 1$.

Statement 3. For each $i \geq 1, \leftrightarrow_{P_{i}}^{*} \subseteq \leftrightarrow_{P_{i+1}}^{*} \subseteq \leftrightarrow_{E}^{*}$.

We can show the following result similarly to Lemma 4.

Lemma 7. For each $i \geq 1$, we can effectively construct the GTES $P_{i}$.

Lemma 8. For each $i \geq 1, \operatorname{sub}\left(p \downarrow_{R_{P_{i}}}\right) \cup\left(\operatorname{sub}\left(R_{P_{i}}\right)-\operatorname{lhs}\left(R_{P_{i}}\right)\right) \cup\left\{t \downarrow_{R_{P_{i}}} \mid\right.$ height $(t) \leq$ $i\} \subseteq N O R M_{i}$.

Proof. By induction on $i$.

We now present our semi-decision procedure.

Procedure PRO4 Input: A variable preserving TES $E$ over the ranked alphabet $\Sigma$ and ground terms $p, q \in T_{\Sigma}$.

Output: • 'yes' if $p \leftrightarrow \leftrightarrow_{E}^{*} q$,

- 'no' if $(p, q) \notin \leftrightarrow_{E}^{*}$ and the procedure halts,

- undefined if the procedure does not halt.

compute $P_{1}, R_{P_{1}}, Q_{1}, R_{Q_{1}}$, and $R_{P_{1} \cup Q_{1}}$;

if $p \downarrow_{R_{P_{1} \cup Q_{1}}}=q \downarrow_{R_{P_{1} \cup Q_{1}}}$, then begin output 'yes'; halt end;

$i:=1$;

$1: i:=i+1$;

compute $P_{i}, R_{P_{i}}, Q_{i}, R_{Q_{i}}$, and $R_{P_{i} \cup Q_{i}}$

if $p \downarrow_{R_{P_{i} \cup Q_{i}}}=q \downarrow_{R_{P_{i} \cup Q_{i}}}$, then begin output 'yes'; halt end; 
if $i=2$, then goto 1 ;

if $R_{P_{i-2}}=R_{P_{i-1}}=P_{i}$, or $R_{Q_{i-2}}=R_{Q_{i-1}}=Q_{i}$,

then begin output 'no'; halt end;

goto 1

Example 17. We continue Example 15. By Statement 3 and (11), $p \downarrow_{R_{P_{i} \cup Q_{i}}} \neq$ $q \downarrow_{R_{P_{i} \cup Q_{i}}}$ for $i \geq 1$. Hence procedure PRO4 does not output 'yes'. By (12) and (13), procedure $\mathrm{PRO}_{4}$ does not output 'no'. Hence procedure $\mathrm{PRO}_{4}$ does not output anything and does not halt at all.

Example 18. We continue Example 16. Observe that

$$
\begin{aligned}
& p \downarrow_{R_{P_{1} \cup Q_{1}}}=0 \neq 1=q \downarrow_{R_{P_{1} \cup Q_{1}}}, \\
& p \downarrow_{R_{P_{2}} \cup Q_{2}}=0 \neq 1=q \downarrow_{R_{P_{2}} \cup Q_{2}}, \\
& p \downarrow_{R_{P_{3}} \cup Q_{3}}=0 \neq 1=q \downarrow_{R_{P_{3}} \cup Q_{3}}, \text { and } \\
& R_{P_{1}}=R_{P_{2}}=P_{3} .
\end{aligned}
$$

Hence procedure $\mathrm{PRO}_{4}$ outputs 'no' and halts in the third step.

Example 19. Let $\Sigma=\Sigma_{0} \cup \Sigma_{1} \cup \Sigma_{2}, \Sigma_{0}=\{\$, \#\}, \Sigma_{1}=\{f, g\}, \Sigma_{2}=\{h\}$. Consider the TES $E=\left\{f f x_{1} \approx g f x_{1}, h\left(x_{1}, x_{1}\right) \approx \$\right\}$. As in Example 2, we can show that the basic Knuth-Bendix completion procedure runs forever on this example. Moreover, it is still open whether the goal-directed completion procedure halts on the TES $E$ and any goal.

Let $n \geq 1$. Let $p=h\left(f^{n} \$, g f^{n-1} \$\right)$ and $q=\$$. We raise the problem whether $p \leftrightarrow{ }_{E}^{*} q$. We now apply procedure PRO4 to the TES $E$ and the terms $p, q$.

GTRS $R_{P_{1}}$ consists of the rules

$$
\begin{aligned}
& f^{i} \$ \rightarrow g f^{i-1} \$ \text { for } 2 \leq i \leq n, \\
& h(\$, \$) \rightarrow \$, \\
& h(\#, \#) \rightarrow \$ .
\end{aligned}
$$

GTRS $R_{Q_{1}}$ consists of the rules

$h(\$, \$) \rightarrow \$$,

$h(\#, \#) \rightarrow \$$.

GTRS $R_{P_{2}}$ consists of the rules

$$
\begin{aligned}
& f^{2} g f \$ \rightarrow g f \$, \\
& h(\$, \$) \rightarrow \$, \\
& h(\#, \#) \rightarrow \$ . \\
& h(f \$, f \$) \rightarrow \$, \\
& h(f \#, f \#) \rightarrow \$ . \\
& h(g \$, g \$) \rightarrow \$, \\
& h(g \#, g \#) \rightarrow \$ .
\end{aligned}
$$

GTRS $R_{Q_{2}}$ consists of the rules

$$
\begin{aligned}
& h(\$, \$) \rightarrow \$, \\
& h(\#, \#) \rightarrow \$, \\
& h(f \$, f \$) \rightarrow \$, \\
& h(f \#, f \#) \rightarrow \$ . \\
& h(g \$, g \$) \rightarrow \$, \\
& h(g \#, g \#) \rightarrow \$ .
\end{aligned}
$$


Clearly,

$p \downarrow_{R_{P_{2}} \cup Q_{2}}=q \downarrow_{R_{P_{2}} \cup Q_{2}}$.

Hence procedure $\mathrm{PRO}_{4}$ outputs 'yes' and halts in the second step.

We now show the correctness of Procedure PRO4.

Lemma 9. Assume that $R_{i-1}=R_{i}=P_{i+1}$ and $N O R M_{i-1} \subset N O R M_{i}$ for some $i \geq 2$. Then for each equation $l \approx r$ of $E \cup E^{-1}$ with $l \in T_{\Sigma}\left(X_{k+m}\right), r \in$ $T_{\Sigma}\left(X_{k} \cup X_{[k+m+1, k+m+\ell]}\right), k, m \geq 0, \ell \geq 1$, and for any $u_{1}, \ldots, u_{k+m} \in \operatorname{sub}\left(p \downarrow_{R_{i}}\right.$ )$\cup\left(\operatorname{sub}\left(R_{i}\right)-l h s\left(R_{i}\right)\right), R_{i}$ does not reach $p \downarrow_{R_{i}}$ starting from $l\left[u_{1}, \ldots, u_{k+m}\right] \downarrow_{R_{i}}$ attached to some context.

Proof. By contradiction. Assume that there is an equation $l \approx r$ of $E \cup E^{-1}$ with $l \in T_{\Sigma}\left(X_{k+m}\right), r \in T_{\Sigma}\left(X_{k} \cup X_{[k+m+1, k+m+\ell]}\right), k, m \geq 0, \ell \geq 1$, and there are $u_{1}, \ldots, u_{k+m} \in \operatorname{sub}\left(p \downarrow_{R_{i}}\right) \cup\left(\operatorname{sub}\left(R_{i}\right)-l h s\left(R_{i}\right)\right)$ such that $R_{i}$ reaches $p \downarrow_{R_{i}}$ starting from $l\left[u_{1}, \ldots, u_{k+m}\right] \downarrow_{R_{i}}$ attached to some context. By $R_{i}=P_{i+1}$, we do not put equations in $P_{i+1}$ in item (b) of its definition. Consequently, for any $v_{k+m+1}, \ldots$, $v_{k+m+\ell} \in N O R M_{i}$,

$$
l\left[u_{1}, \ldots, u_{k+m}\right] \downarrow_{R_{i}}=r\left[u_{1}, \ldots, u_{m}, v_{k+m+1}, \ldots, v_{k+m+\ell}\right] \downarrow_{R_{i}} .
$$

Hence by our indirect assumption, $R_{i}$ reaches $p_{\downarrow_{i}}$ starting from $r\left[u_{1}, \ldots, u_{m}, v_{k+m+1}, \ldots, v_{k+m+\ell}\right] \downarrow_{R_{i}}$ attached to some context. Hence there is a $u \in C_{\Sigma}$ such that

$$
u\left[r\left[u_{1}, \ldots, u_{m}, v_{k+m+1}, \ldots, v_{k+m+\ell}\right] \downarrow_{R_{i}}\right] \stackrel{*}{\underset{R}{\rightarrow}} p \downarrow_{R_{i}} .
$$

Then $u\left[r\left[u_{1}, \ldots, u_{m}, v_{k+m+1} \downarrow_{R_{i}}, v_{k+m+2}, \ldots, v_{k+m+\ell}\right]\right] \rightarrow_{R_{i}}^{*}$

$u\left[r\left[u_{1}, \ldots, u_{m}, v_{k+m+1}, \ldots, v_{k+m+\ell}\right] \downarrow_{R_{i}}\right] \rightarrow_{R_{i}}^{*} p \downarrow_{R_{i}}$. By Lemma $2, v_{k+m+1} \downarrow_{R_{i}} \in$ $\operatorname{sub}\left(p \downarrow_{R_{i}}\right) \cup\left(\operatorname{sub}\left(R_{i}\right)-l h s\left(R_{i}\right)\right)$. Since $R_{i-1}=R_{i}$,

$v_{k+m+1} \downarrow_{R_{i-1}} \in \operatorname{sub}\left(p \downarrow_{R_{i-1}}\right) \cup\left(\operatorname{sub}\left(R_{i-1}\right)-\operatorname{lhs}\left(R_{i-1}\right)\right) \subseteq N O R M_{i-1}$.

By definition, $v_{k+m+1}$ is an arbitrary element of $N O R M_{i}$. Consequently, we have $N_{O R} M_{i} \subseteq N O R M_{i-1}$. This is a contradiction.

Lemma 10. Let $i \geq 2$. If $R_{i-1}=R_{i}=R_{i+1}$ and $N O R M_{i-1}=N O R M_{i}$, then $N O R M_{i}=N O R M_{i+1}$.

Proof. First we show that $N O R M_{i} \subseteq N O R M_{i+1}$. Let $s \in N O R M_{i}$ be arbitrary. If $s \in \operatorname{sub}\left(p \downarrow_{R_{i}}\right) \cup\left(\operatorname{sub}\left(R_{i}\right)-\operatorname{lhs}\left(R_{i}\right)\right) \cup\left\{t \downarrow_{R_{i}} \mid t \in N O R M_{i-1}\right\}$, then $s \in \operatorname{sub}\left(p \downarrow_{R_{i+1}}\right) \cup\left(\operatorname{sub}\left(R_{i+1}\right)-\operatorname{lhs}\left(R_{i+1}\right)\right) \cup\left\{t \downarrow_{R_{i+1}} \mid t \in N O R M_{i}\right\}$. Hence $t \in$ $N O R M_{i+1}$. If $s=f\left(t_{1}, \ldots, t_{m}\right) \downarrow_{R_{i}}$ for some $f \in \Sigma_{m}$ and $t_{1}, \ldots, t_{m} \in N O R M_{i-1}$, then $s=f\left(t_{1}, \ldots, t_{m}\right) \downarrow_{R_{i+1}}$ with $f \in \Sigma_{m}$ and $t_{1}, \ldots, t_{m} \in N O R M_{i}$. Hence $t \in N O R M_{i+1}$.

We now show that $N O R M_{i+1} \subseteq N O R M_{i}$. Let $s \in N O R M_{i+1}$ be arbitrary. If $s \in \operatorname{sub}\left(p \downarrow_{R_{i+1}}\right) \cup\left(\operatorname{sub}\left(R_{i+1}\right)-\operatorname{lhs}\left(R_{i+1}\right)\right) \cup\left\{t \downarrow_{R_{i+1}} \mid t \in N O R M_{i}\right\}$, then $s \in \operatorname{sub}\left(p \downarrow_{R_{i}}\right) \cup\left(\operatorname{sub}\left(R_{i}\right)-\operatorname{lhs}\left(R_{i}\right)\right) \cup\left\{t \downarrow_{R_{i}} \mid t \in N O R M_{i-1}\right\}$. Hence $t \in N O R M_{i}$. 
If $s=f\left(t_{1}, \ldots, t_{m}\right) \downarrow_{R_{i+1}}$ for some $f \in \Sigma_{m}$ and $t_{1}, \ldots, t_{m} \in N O R M_{i}$, then $s=$ $f\left(t_{1}, \ldots, t_{m}\right) \downarrow_{R_{i}}$ for $f \in \Sigma_{m}$ and $t_{1}, \ldots, t_{m} \in N O R M_{i-1}$. Hence $t \in N O R M_{i}$.

Lemma 11. For each $i \geq 2$, if $R_{i-1}=R_{i}=P_{i+1}$, then $R_{i}=R_{i+1}=P_{i+2}$.

Proof. By the assumption $R_{i}=P_{i+1}$ and the definition of $R_{i+1}$, we have

$$
R_{i}=R_{i+1}
$$

We now distinguish two cases.

Case 1: $N O R M_{i-1}=N O R M_{i}$. By Lemma 10,

$$
\operatorname{NORM}_{i}=\operatorname{NORM}_{i+1} .
$$

By (14) and (15), $P_{i+1}=P_{i+2}$. By the assumption $R_{i}=P_{i+1}$ and (14), we have $R_{i}=R_{i+1}=P_{i+2}$.

Case 2: $\operatorname{NORM}_{i-1} \subset N O R M_{i}$. Then by Lemma 9, for each equation $l \approx r$ of $E \cup E^{-1}$ with $l \in T_{\Sigma}\left(X_{k+m}\right), r \in T_{\Sigma}\left(X_{k} \cup X_{[k+m+1, k+m+\ell]}\right), k, m \geq 0, \ell \geq 1$, and for any $u_{1}, \ldots, u_{k+m} \in\left(\operatorname{sub}\left(R_{i}\right)-l h s\left(R_{i}\right)\right) \cup \operatorname{sub}\left(p \downarrow_{R_{i}}\right), R_{i}$ does not reach $p \downarrow_{R_{i}}$ starting from $l\left[u_{1}, \ldots, u_{k+m}\right] \downarrow_{R_{i}}$ attached to some context. Then by (14), we do not put equations in $P_{i+2}$ in item (b) in the definition of $P_{i+2}$. Hence $R_{i+1}=P_{i+2}$. By (14) the proof is complete.

Lemma 11 implies the following.

Lemma 12. For each $i \geq 1$, if $R_{i-1}=R_{i}=P_{i+1}$, then for each $k \geq 1, R_{i}=$ $R_{i+k}=P_{i+k+1}$.

We now show the correctness of Procedure $\mathrm{PRO}_{4}$.

Lemma 13. For any $n \geq 1, t_{1}, \ldots, t_{n} \in T_{\Sigma}$, if $p \leftrightarrow_{E} t_{1} \leftrightarrow_{E} t_{2} \leftrightarrow_{E} \cdots \leftrightarrow_{E} t_{n}$, then there is $i \geq 1$ such that $p \leftrightarrow \leftrightarrow_{P_{i}}^{*} t_{1} \leftrightarrow \stackrel{P}{i}^{*} t_{2} \leftrightarrow_{P_{i}}^{*} \cdots \leftrightarrow \leftrightarrow_{P_{i}}^{*} t_{n}$.

Proof. We proceed by induction on $n$.

Base Case: $n=1$. Assume that $p \leftrightarrow_{E} t_{1}$. Then there is an equation $l \approx r$ of $E \cup E^{-1}$ with $l \in T_{\Sigma}\left(X_{k+m}\right), r \in T_{\Sigma}\left(X_{k+m+\ell}\right), k, m, \ell \geq 0$, and there is $u \in C_{\Sigma}$, $u_{1}, \ldots, u_{k+m}, v_{k+m+1}, \ldots, v_{k+m+\ell} \in T_{\Sigma}$ such that

$$
p=u\left[l\left[u_{1}, \ldots, u_{k+m}\right]\right]
$$

and $t_{1}=u\left[r\left[u_{1}, \ldots, u_{k}, v_{k+m+1}, \ldots, v_{k+m+\ell}\right]\right]$.

Let $i=\max \left\{h e i g h t\left(v_{k+1}\right), \ldots\right.$, height $\left.\left(v_{k+m+\ell}\right)\right\}$. By Lemma $8, v_{k+m+1} \downarrow_{R_{i}}, \ldots$, $v_{k+m+\ell} \downarrow_{R_{i}}$ are in $N O R M_{i}$. By $(16), R_{i}$ reaches $p \downarrow_{R_{i}}$ from $l\left[u_{1} \downarrow_{R_{i}}, \ldots, u_{k+m} \downarrow_{R_{i}}\right] \downarrow_{R_{i}}$ attached to some context. By the definition of $P_{i+1}$, the equation

$$
l\left[u_{1} \downarrow_{R_{i}}, \ldots, u_{k+m} \downarrow_{R_{i}}\right] \approx r\left[u_{1} \downarrow_{R_{i}}, \ldots, u_{k \downarrow}{R_{i}}_{i}, v_{k+m+1} \downarrow_{R_{i}}, \ldots, v_{k+m+\ell} \downarrow_{R_{i}}\right]
$$

is in $\leftrightarrow_{P_{i}}^{*}$ or $P_{i+1}$. Hence, by the definition of $R_{i}$ and Statement 3 , 


$$
\begin{aligned}
& p=u\left[l\left[u_{1}, \ldots, u_{k+m}\right]\right] \leftrightarrow_{P_{i+1}}^{*} u\left[l\left[u_{1} \downarrow_{R_{i}}, \ldots, u_{k+m} \downarrow R_{i}\right]\right] \leftrightarrow_{P_{i+1}}^{*} \\
& u\left[r\left[u_{1} \downarrow R_{i}, \ldots, u_{k} \downarrow R_{i}, v_{k+m+1} \downarrow R_{i}, \ldots, v_{k+m+\ell}\right] \downarrow R_{i}\right] \leftrightarrow_{P_{i}}^{*} \\
& u\left[r\left[u_{1}, \ldots, u_{k}, v_{k+m+1}, \ldots, v_{k+m+\ell}\right]\right]=t_{1} .
\end{aligned}
$$

Then we have $p \leftrightarrow{ }_{P_{i+1}}^{*} t_{1}$.

Induction Step: Let $n \geq 1$, and assume that the satement holds for $1,2, \ldots, n$. We now show that the satement holds for $n+1$. To this end, assume that

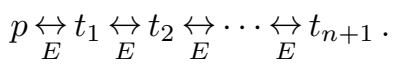

By the induction hypothesis, there is $j \geq 1$ such that

$$
p \stackrel{*}{\stackrel{*}{\leftrightarrow}} t_{1} \underset{P_{j}}{\stackrel{*}{\leftrightarrow}} t_{2} \underset{P_{j}}{\stackrel{*}{\leftrightarrow}} \cdots \stackrel{*}{\leftrightarrow} t_{P_{j}}
$$

Hence

$$
t_{n} \underset{R_{i}}{\stackrel{*}{\rightarrow}} p \downarrow_{R_{i}} .
$$

By (17), there is an equation $l \approx r$ in $E \cup E^{-1}$ with $l \in T_{\Sigma}\left(X_{k+m}\right), r \in T_{\Sigma}\left(X_{k} \cup\right.$ $\left.X_{[k+m+1, k+m+\ell]}\right)$ for some $k, m, \ell \geq 0$, and there are $u \in C_{\Sigma}$,

$u_{1}, \ldots, u_{k+m}, v_{k+m+1}, \ldots, v_{k+m+\ell} \in T_{\Sigma}$ such that

$$
t_{n}=u\left[l\left[u_{1}, \ldots, u_{k+m}\right]\right] \text { and } t_{n+1}=u\left[r\left[u_{1}, \ldots, u_{k}, v_{k+m+1}, \ldots, v_{k+m+\ell}\right]\right] .
$$

Let $i=\max \left\{j, h e i g h t\left(v_{k+m+1}\right), \ldots\right.$,height $\left.\left(v_{k+m+\ell}\right)\right\}$. By Lemma 8, $v_{k+m+1} \downarrow_{R_{i}}$ $, \ldots, v_{k+m+\ell} \downarrow_{R_{i}}$ are in NORM $M_{i}$. Clearly, $l\left[u_{1} \downarrow_{R_{i}}, \ldots, u_{k+m} \downarrow_{R_{i}}\right] \rightarrow_{R_{i}}^{*} l\left[u_{1} \downarrow_{R_{i}}\right.$ $\left., \ldots, u_{k+m} \downarrow R_{i}\right] \downarrow_{R_{i}}$. Then by (19) and (20), $R_{i}$ reaches $p \downarrow_{R_{i}}$ starting from $l\left[u_{1} \downarrow_{R_{i}}\right.$ $\left., \ldots, u_{k+m} \downarrow_{R_{i}}\right] \downarrow_{R_{i}}$ attached to some context. By the definition of $P_{i+1}$, the equation

$l\left[u_{1} \downarrow_{R_{i}}, \ldots, u_{k+m} \downarrow_{R_{i}}\right] \approx r\left[u_{1} \downarrow_{R_{i}}, \ldots, u_{k} \downarrow_{R_{i}}, v_{k+m+1} \downarrow_{R_{i}}, \ldots, v_{k+m+\ell} \downarrow_{R_{i}}\right]$

is in $\leftrightarrow_{P_{i}}^{*}$ or $P_{i+1}$. Hence, by the definition of $R_{i}$ and Statement 3,

$t_{n}=u\left[l\left[u_{1}, \ldots, u_{k+m}\right]\right] \leftrightarrow_{P_{i+1}}^{*} u\left[l\left[u_{1} \downarrow_{R_{i}}, \ldots, u_{k+m} \downarrow_{R_{i}}\right]\right] \leftrightarrow_{P_{i+1}}$

$u\left[r\left[u_{1} \downarrow_{R_{i}}, \ldots, u_{k} \downarrow_{R_{i}}, v_{k+m+1} \downarrow_{R_{i}}, \ldots, v_{k+m+\ell} \downarrow_{R_{i}}\right]\right] \leftrightarrow_{P_{i+1}}^{*}$

$u\left[r\left[u_{1}, \ldots, u_{k}, v_{k+m+1}, \ldots, v_{k+m+\ell}\right]\right]=t_{n+1}$.

By (18), $p \leftrightarrow_{P_{i+1}}^{*} t_{1} \leftrightarrow_{P_{i+1}}^{*} t_{2} \leftrightarrow_{P_{i+1}}^{*} \cdots \leftrightarrow_{P_{i+1}}^{*} t_{n} \leftrightarrow_{P_{i+1}}^{*} t_{n+1}$.

By Statement 3, Lemma 12, and Lemma 13 we have the following result.

Lemma 14. For each $i \geq 2$, if $R_{i-1}=R_{i}=P_{i+1}$, then for each $q^{\prime} \in T_{\Sigma}, p \leftrightarrow_{P_{i}}^{*} q^{\prime}$ if and only if $p \leftrightarrow{ }_{E}^{*} q^{\prime}$.

We can show the following in the same way as Theorem 1.

Theorem 5. If $p \leftrightarrow_{E}^{*} q$, then procedure PRO4 outputs 'yes' and halts.

We can show the following in the same way as Theorem 2.

Theorem 6. If procedure PRO4 outputs 'yes' and halts, then $p \leftrightarrow_{E}^{*} q$. If procedure PRO4 outputs 'no' and halts, then $(p, q) \notin \leftrightarrow_{E}^{*}$.

Theorems 5 and 6 imply the following.

Theorem 7. If $p \leftrightarrow_{E}^{*} q$, then procedure PRO4 outputs 'yes' and halts. Otherwise, either PRO4 outputs 'no' and halts, or PRO4 does not halt at all. 


\section{Comparison with the Knuth-Bendix completion procedure}

We now compare procedures $\mathrm{PRO}_{3}$ and $\mathrm{PRO}_{4}$ with the basic Knuth-Bendix completion procedure (see Section 7.1 in [1]), the improved version of the Knuth-Bendix completion procedure described by a set of inference rules (see Section 7.2 in [1]), the goal-directed completion procedure based on SOUR graphs [13, 14], and the unfailing Knuth-Bendix completion procedure [2]. In contrast to all versions of the Knuth-Bendix procedure, Procedures PRO3 and PRO4 do not compute any critical pairs and do not use a reduction order. They do not attempt to construct a convergent TRS equivalent to $E$. When $\mathrm{PRO}_{3}$ and $\mathrm{PRO}_{4}$ run a congruence closure algorithm for the TES $E$ over the subterm graph of $E[4,15]$, they compute and then process only finitely many ground instances $(\bar{s}, \bar{t})$ of finitely many elements $(s, t)$ of the relation $\leftrightarrow_{E}^{*}$, where $s, t$ may contain variables. Here $(s, t)$ need not be a critical pair computed by the basic Knuth-Bendix completion procedure. In fact, the ground instances $(\bar{s}, \bar{t})$ are elements of the equivalence relation $\leftrightarrow_{E}^{*} \cap(\operatorname{sub}(E) \times \operatorname{sub}(E))$. Procedures $\mathrm{PRO}_{3}$ and $\mathrm{PRO}_{4}$ compute a representative $r$ of $\bar{s}$ and $\bar{t}$ for the equivalence relation $\leftrightarrow_{E}^{*} \cap(s u b(E) \times s u b(E))$. The representative $r$ becomes the normal form of $\bar{s}$ and $\bar{t}$ for the rewrite relation induced by the constructed reduced GTRS. Hence, PRO3 and PRO4 do not compare the normal forms of $s$ and $t$ via any reduction order. In contrast, the basic Knuth-Bendix completion procedure reduces the terms in each critical pair to their normal forms. Then tries to orient the normal forms into a rewrite rule. In this way the procedure orients all instances of these terms as well. The improved version of the Knuth-Bendix completion procedure described by a set of inference rules (see Section 7.2 in [1]) also processes each critical pair and also orients the obtained pair, and hence all of its instances. The unfailing Knuth-Bendix completion procedure [2] applies orientable instances of equations in $E$ with respect to a reduction order $>$.

To illustrate the efficiency of the goal-directed completion procedure, Lynch [13] presented the following example. Let the ranked alphabet $\Sigma$ consist of the unary symbols $f, g$ and the nullary symbols $\$$, \#. Consider the variable preserving TES $E=\{f f x \approx g f x\}$. We raise the problem whether $\$ \leftrightarrow_{E}^{*} \#$. On the one hand, the basic Knuth-Bendix completion procedure runs forever on this example [13]. On the other hand, the goal-directed completion procedure does not generate any rule applicable to $\$$ or \#. Therefore, the goal-directed completion procedure outputs 'no' and halts [13]. Lynch and Strogova [14] said that "the goal-directed completion procedure compiles the TES $E$ and the goal $(p, q)$. After the compilation is finished, we cannot apply a schematization of an equation in the completed system. Therefore, the goal-directed completion procedure outputs 'no' and halts. This is an example where the goal-directed completion procedure is superior to the basic Knuth-Bendix algorithm." It is still open whether the goal-directed completion procedure halts on the TES $E$ and any goal [13]. As for the above example, PRO3 gives the correct answer and then halts on the TES $E$ and any terms $p, q \in T_{\Sigma}$.

We conjecture that there are variable preserving TES $E$ and ground terms $p, q$ 
such that Conditions (a)-(c) hold.

(a) The basic Knuth-Bendix completion procedure runs forever on $E$.

(b) There is a goal $(p, q)$ such that the goal-directed completion procedure does not stop on $E$ and $(p, q)$.

(c) Procedure PRO3 gives the correct answer and then halts on the TES $E$ and any terms $p, q \in T_{\Sigma}$.

Let TES $E$ be as in Example 11. We conjecture that there is $q \in T_{\Sigma}$ such that the symbols $a, c$ do not appear in $q$ and that the goal-directed completion procedure does not halt on the TES $E$ and the goal (aaa\$,q). On the other hand, let $q \in T_{\Sigma}$ be arbitrary such that the symbols $a, c$ do not appear in $q$. On the input $E, a a a \$$, q, Procedure PRO3 outputs 'no', the correct answer, and then halts, see Example 11.

Procedures $\mathrm{PRO}_{3}$ and $\mathrm{PRO}_{4}$ attempt to construct the reduced GTRSs $R_{P}$ and $R_{Q}$, rather than a convergent term rewrite system equivalent to $E$, such that

- $R_{P} \cup R_{Q} \subseteq \leftrightarrow_{E}^{*}$,

- $p \leftrightarrow_{R_{P}}^{*} q$ or $\leftrightarrow_{R_{P}}^{*} \cap\left(\{p\} \times T_{\Sigma}\right)=\leftrightarrow_{E}^{*} \cap\left(\{p\} \times T_{\Sigma}\right)$, and

- $p \leftrightarrow_{R_{Q}}^{*} q$ or $\leftrightarrow_{R_{Q}}^{*} \cap\left(\{q\} \times T_{\Sigma}\right)=\leftrightarrow_{E}^{*} \cap\left(\{q\} \times T_{\Sigma}\right)$.

Thus $R_{P}$ and $R_{Q}$ need not be equivalent to $E$. By contrast, all versions of the Knuth-Bendix completion procedure attempt to transform a given TES $E$ into an equivalent convergent term rewrite sytem. Since Snyder's ground completion algorithm does not apply orderings, procedures $\mathrm{PRO}_{3}$ and $\mathrm{PRO}_{4}$ do not apply any orderings as well.

We now present three examples where procedures $\mathrm{PRO} 3$ and $\mathrm{PRO} 4$ compute efficiently, probably more efficiently than all versions of the Knuth-Bendix completion procedure.

Example 20. $[8,16]$ Gallier et al [8] and Plaisted and Sattler-Klein [16] presented the following problem to illustrate that reducing a ground term to its normal form can take exponential time if a proper strategy is not used. Let $\Sigma=\Sigma_{0} \cup \Sigma_{1}$, $\Sigma_{0}=\{\$\}$, and $\Sigma_{1}=\{f, g\}$. Let $n \geq 2$. Let the GTRS $R$ consist of the following rules:

$$
\begin{aligned}
& f \$ \rightarrow g \$, \\
& f g \$ \rightarrow g f \$, \\
& f g^{2} \$ \rightarrow g f^{2} \$, \\
& \cdots \\
& f g^{n} \$ \rightarrow g f^{n} \$ .
\end{aligned}
$$

Plaisted and Sattler-Klein observed the following on page 156 in [16]. Although GTRS $R$ is convergent, the right-hand sides can be further rewritten. An unskilful choice of rewrites can lead to an exponential time of process. The straightforward reduction of the term $g f^{n} \$$ can take a number of rewrite steps exponential in $n$. However, if we apply the rules in order of size, smallest first, to all other rules, the whole TRS can be rewritten to a reduced GTRS in a polynomial number of steps.

We form the TES $E$ by adding the equation

$$
f g^{n+1} x \approx g f^{n+1} x
$$


to the set $R$. We now run procedure PRO3 on the variable preserving TES $E$ and the ground terms $p=f^{n+2} \$$ and $q=g^{n+2} \$$. Then

$$
\begin{aligned}
& \{f \$ \approx g \$\}=P_{1}=R_{P_{1}}=Q_{1}=R_{Q_{1}}, p \downarrow_{R_{P_{1} \cup Q_{1}}} \neq q \downarrow_{R_{P_{1} \cup Q_{1}}}=q . \\
& R_{1} \cup\left\{f g \$ \approx g^{2} \$\right\}=P_{2}=R_{P_{2}}=Q_{2}=R_{Q_{2}}, p \downarrow_{R_{P_{2}} \cup Q_{2}} \neq q \downarrow_{R_{P_{2} \cup Q_{2}}}=q . \\
& R_{2} \cup\left\{f g^{2} \$ \approx g^{3} \$\right\}=P_{3}=R_{P_{3}}=Q_{3}=R_{Q_{3}}, p \downarrow_{R_{P_{3}} \cup Q_{3}} \neq q \downarrow_{R_{P_{3}} \cup Q_{3}}=q . \\
& \ldots \\
& R_{P_{n}} \cup\left\{f g^{n} \$ \approx g^{n} \$\right\}=P_{n}=R_{P_{n}}=Q_{n}=R_{Q_{n}}, p \downarrow_{R_{P_{n+1}} \cup Q_{n+1}} \neq q \downarrow_{R_{P_{n+1} \cup Q_{n+1}}} . \\
& R_{P_{n+1}} \cup\left\{f g^{n+1} \$ \approx g^{n+1} \$\right\}=P_{n+2}=R_{P_{n+2}}=Q_{n+2}=R_{Q_{n+2}} . \\
& P_{n+2}=R_{P_{n+3}}=Q_{n+2}=R_{Q_{n+3}} .
\end{aligned}
$$

Observe that $p \downarrow_{R_{P_{n+2}} \cup Q_{n+2}}=q \downarrow_{R_{P_{n+2} \cup Q_{n+2}}}=q$. Hence procedure PRO3 outputs 'yes' and halts in the $(n+2)$ nd step. The number of computation steps is polynomial. It should be clear that for all ground terms $p$ and $q, P R O 3$ halts. It outputs 'yes' if $p \leftrightarrow{ }_{E}^{*} q$. Otherwise it outputs 'no'.

Consider the lexicographic path order $>_{l p o}$ induced by the order $f>g>\$$ [1]. We now run the basic Knuth-Bendix completion procedure on the TES $E$ and the reduction order $>_{\text {lpo }}$. In the initialization phase, the basic Knuth-Bendix completion procedure orients the equations of $E$. We obtain the TRS $S$ consisting of the following rules:

$$
\begin{aligned}
& f \$ \rightarrow g \$, \\
& f g \$ \rightarrow g f \$, \\
& f g^{2} \$ \rightarrow g f^{2} \$, \\
& \cdots \\
& f g^{n} \$ \rightarrow g f^{n} \$, \\
& f g^{n+1} x \rightarrow g f^{n+1} x .
\end{aligned}
$$

Similarly to the first part of the example we have the following. The TRS $S$ has no critical pairs. Hence the basic Knuth-Bendix procedure outputs $S$. The straightforward reduction of the term $f^{n+2} \$$ to $g^{n+2} \$$ by $S$ takes a number of rewrite steps exponential in $n$. The improved Knuth-Bendix completion procedure reduces the right-hand sides of the first $n$ rules as in the first part of the example. We obtain the TRS $S^{\prime}$ consisting of the following rules:

$$
\begin{aligned}
& f \$ \rightarrow g \$, \\
& f g \$ \rightarrow g f \$, f g \$ \rightarrow g g \$, \\
& f g^{2} \$ \rightarrow g f^{2} \$, f g^{2} \$ \rightarrow g f g \$, f g^{2} \$ \rightarrow g^{3} \$, \\
& \cdots \\
& f g^{n} \$ \rightarrow g f^{n} \$, f g^{n} \$ \rightarrow g f^{n-1} g \$, \ldots, f g^{n} \$ \rightarrow g^{n+1} \$, \\
& f g^{n+1} x \rightarrow g f^{n+1} x .
\end{aligned}
$$

In the best case, the reduction of the term $f^{n+2} \$$ to $g^{n+2} \$$ applies the rules

$$
\begin{aligned}
& f \$ \rightarrow g \$, \\
& f g \$ \rightarrow g g \$, \\
& f g^{2} \$ \rightarrow g^{3} \$, \\
& \cdots \\
& f g^{n} \$ \rightarrow g^{n+1} \$, \\
& f g^{n+1} x \rightarrow g f^{n+1} x .
\end{aligned}
$$


In the worst case, $S^{\prime}$ applies only the rules of $S$ in the reduction of the term $f^{n+2} \$$ to $g^{n+2} \$$. Hence it takes a number of rewrite steps exponential in $n$ as in the first part of the example. The goal-directed completion procedure computes fast on $E$ and the goal $(p, q)$. For experimental results, see the line of the problem Counter5 in Table 1 in Section 7 in [14].

Example 21. We now modify an example of Plaisted and Sattler-Klein [16] and Lynch and Strogova [14].

Let $n \geq 2, \Sigma=\Sigma_{0} \cup \Sigma_{2}, \Sigma_{0}=\left\{\$_{1}, \$_{2}, \ldots, \$_{n}, \#_{1}, \#_{2}, \ldots, \#_{n}\right\}$, and $\Sigma_{2}=$ $\{f, g\}$. Let the TES $E$ consist of the following equations:

$f(b, b) \approx f\left(\#_{0}, \$_{0}\right)$,

$\$_{0} \approx f\left(\$_{1}, \#_{1}\right)$,

$\#_{0} \approx g\left(\#_{1}, \$_{1}\right)$,

$\$_{1} \approx f\left(\$_{2}, \# 2\right)$

$\#_{1} \approx g\left(\#_{2}, \$_{2}\right)$

...

$\$_{n-1} \approx f\left(\$_{n}, \#_{n}\right)$

$\#_{n-1} \approx g\left(\#_{n}, \$_{n}\right)$,

$\$_{n} \approx \#_{n}$,

$f\left(x_{1}, x_{1}\right) \approx g\left(x_{1}, x_{1}\right)$.

We now run procedure $P R O 3$ on the variable preserving TES $E$ and the ground terms $p=f\left(\$_{0}, \#_{0}\right)$ and $q=g\left(\#_{0}, \#_{0}\right)$. Then

$\left\{f\left(\$_{1}, \#_{1}\right) \approx \$_{0}, g\left(\#_{1}, \$_{1}\right) \approx \#_{0}\right\}=P_{1}=R_{P_{1}}$,

$\left\{g\left(\#_{1}, \$_{1}\right) \approx \#_{0}, f\left(\#_{0}, \#_{0}\right) \approx g\left(\#_{0}, \#_{0}\right)\right\}=Q_{1}=R_{Q_{1}}$,

$R_{P_{1}} \cup\left\{f\left(\$_{2}, \#_{2}\right) \approx \$_{1}, g\left(\#_{2}, \$_{2}\right) \approx \$_{1}\right\}=P_{2}=R_{P_{2}}$,

$R_{Q_{1}} \cup\left\{f\left(\$_{1}, \#_{1}\right) \approx \$_{0}, f\left(\$_{2}, \#_{2}\right) \approx \$_{1}, g\left(\#_{2}, \$_{2}\right) \approx \#_{1}\right\}=Q_{2}=R_{Q_{2}}$,

...

$R_{P_{n-1}} \cup\left\{f\left(\$_{n}, \#_{n}\right) \approx \$_{n-1}, g\left(\#_{n}, \$_{n}\right) \approx \#_{n-1}\right\}=P_{n}=R_{P_{n}}$

$R_{Q_{n-1}} \cup\left\{f\left(\$_{n-1}, \#_{n-1}\right) \approx \$_{n-2}, g\left(\#_{n}, \$_{n}\right) \approx \#_{n-1}\right\}=Q_{n}=R_{Q_{n}}$.

$R_{P_{n}}$ consists of the following rules:

$f\left(\$_{1}, \#_{1}\right) \rightarrow \$_{0}$

$g\left(\#_{1}, \$_{1}\right) \rightarrow \#_{0}$

$f\left(\$_{2}, \#_{2}\right) \rightarrow \$_{1}$,

$g\left(\#_{2}, \$_{2}\right) \rightarrow \#_{1}$

$f\left(\$_{n}, \#_{n}\right) \rightarrow \$_{n-1}$,

$g\left(\#_{n}, \$_{n}\right) \rightarrow \#_{n-1}$,

$R_{P_{n}} \cup\left\{\$_{n} \approx \#_{n}\right\}=P_{n+1}$.

$R_{P_{n+1}}$ consists of the following rules:

$f\left(\$_{0}, \#_{0}\right) \rightarrow \$_{0}$,

$f\left(\$_{1}, \$_{1}\right) \rightarrow \$_{0}$,

$f\left(\$_{2}, \$_{2}\right) \rightarrow \$_{1}$,

$g\left(\$_{n}, \$_{n}\right) \rightarrow \$_{n-1}$,

$\#_{0} \rightarrow \$_{0}$, 


$$
\begin{aligned}
& \#_{1} \rightarrow \$_{1}, \\
& \#_{2} \rightarrow \$_{2}, \\
& \ldots \\
& \#_{n} \rightarrow \$_{n} . \\
& R_{Q_{n}} \cup\left\{f\left(\$_{n}, \#_{n}\right) \approx \$_{n-1}, \$ \approx \#\right\}=Q_{n+1}, \\
& R_{Q_{n+1}}=R_{P_{n+1}} \cup\left\{f\left(\#_{0}, \#_{0}\right) \rightarrow g\left(\#_{0}, \#_{0}\right)\right\} . \\
& P_{n+2}=R_{P_{n+3}}=Q_{n+2}=R_{Q_{n+3}} .
\end{aligned}
$$

Clearly, $p \downarrow_{R_{P_{n+1}}}=q \downarrow_{R_{P_{n+1}}}$. Consequently, procedure PRO3 outputs 'yes' and halts in the $(n+1)$ st step. The number of computation steps is polynomial.

Consider the lexicographic path order $>_{l p o}$ induced by the order

$$
b>\$_{0}>\$_{1}>\cdots>\$_{n}>\#_{0}>\#_{1}>\cdots>\#_{n}>f>g .
$$

We now run the basic Knuth-Bendix completion procedure on the TES $E$ and the reduction order $>_{l p o}$. In the initialization phase, the basic Knuth-Bendix completion procedure orients the equations of $E$. We obtain the TRS $S$ consisting of the following rules:

$$
\begin{aligned}
& \$_{0} \rightarrow f\left(\$_{1}, \#_{1}\right), \\
& \#_{0} \rightarrow g\left(\#_{1}, \$_{1}\right), \\
& \$_{1} \rightarrow f\left(\$_{2}, \#_{2}\right), \\
& \#_{1} \rightarrow g\left(\#_{2}, \$_{2}\right), \\
& \ldots \\
& \$_{n-1} \rightarrow f\left(\$_{n}, \#_{n}\right), \\
& \#_{n-1} \rightarrow g\left(\#_{n}, \$_{n}\right), \\
& \$_{n} \rightarrow \#_{n}, \\
& f(b, b) \rightarrow f\left(\#_{0}, \$_{0}\right), \\
& f\left(x_{1}, x_{1}\right) \rightarrow g\left(x_{1}, x_{1}\right) .
\end{aligned}
$$

The last two rules yield the critical pair $\left\langle f\left(\#_{0}, \$_{0}\right), g(b, b)\right\rangle$. Observe that $f\left(\#_{0}, \$_{0}\right)$ has a unique $\rightarrow_{S}$ normal form, and that $\operatorname{size}\left(f\left(\#_{0}, \$_{0}\right) \downarrow_{S}\right)=2^{n+1}$. Thus the completed system contains a rule with a left-hand side of size $2^{n+1}$. The improved Knuth-Bendix completion procedure also yields the TRS $S$ and the above critical pair. Again, the completed system contains a rule with a left-hand side of size $2^{n+1}$. The goal-directed completion procedure based on SOUR graphs $[13,14]$ stores the term $f\left(\#_{0}, \$_{0}\right) \downarrow_{S}$ in linear space in $n$.

Example 22. Let $\Sigma=\Sigma_{0} \cup \Sigma_{1}, \Sigma_{0}=\{\$\}$, and $\Sigma_{1}=\{a, b\}$. Let the GTES $F$ consist of the equation $a_{b b a x} \approx x_{1}$. Furthermore, let the GTES $E$ consist of the equations

$$
\operatorname{abbax}_{1} \approx x_{1}, a \$ \approx \$, b \$ \approx \$ .
$$

It is well-known that there is no convergent TRS $R$ equivalent to $F$, see Theorem 4.2.18 in [10]. Hence there is no convergent TRS $R$ equivalent to $E$ either. Consequently, the basic Knuth-Bendix completion procedure (see Section 7.1 in [1]), the improved version of the Knuth-Bendix completion procedure described by a set of inference rules (see Section 7.2 in [1]) cannot produce a convergent TRS $R$ equivalent to $E$. 
Let $p, q \in T_{\Sigma}$ be arbitrary. First, we run the procedure $P R O 3$ on the input $E, p, q$. Procedure $\mathrm{PRO} 3$ outputs 'yes' and halts in the first or second step. The resulting reduced GTRS is a subset of

$\{a \$ \rightarrow \$, b \$ \rightarrow \$\}$.

Second, we run the goal-directed completion procedure on the input $E,(p, q)$. It computes all critical pairs and then processes them. Then it applies the resulting rules. The goal-directed completion procedure takes more time on $E$ and the goal $(p, q)$ than procedure $P R O 3$ on the input $E, p, q$.

\section{Conclusion}

We recalled the well known trivial semi-decision procedure $P R O 1$ for the ground word problem of variable preserving TESs and its straightforward generalization, the trivial semi-decision procedure PRO2 for the ground word problem of TESs. On the basis of PRO1, we gave the semi-decision procedure PRO3 for the ground word problem of variable preserving TESs. We gave examples when procedure PRO3 was more efficient than procedure PRO1. Then we presented the semi-decision procedure $\mathrm{PRO}_{4}$ for the ground word problem of term equation systems. We obtained it generalizing $P R O 3$ taking into account PRO2. We showed the correctness of $P R O 3$ and $P R O 4$. We compared the procedures $P_{R O} 3$ and $\mathrm{PRO}_{4}$ with the basic Knuth-Bendix completion procedure and the goal-directed completion procedure based on SOUR graphs $[13,14]$.

Procedures $\mathrm{PRO} 3$ or $\mathrm{PRO}_{4}$ compute in a different way than all versions of the Knuth-Bendix completion procedure. To some instances of the ground word problem of a TES $E$, they give an answer sooner than all versions of the Knuth-Bendix completion procedure or it is open whether some version of the Knuth-Bendix completion procedure gives an answer at all. Assume that, given a TES $E$ and ground terms $p, q$, we want to decide whether $p \leftrightarrow_{E}^{*} q$. The ground word problem is undecidable even for variable-preserving TESs. Consequently, we have no upper bound on the running time of any type of the Knuth-Bendix completion procedure on the input TES $E$ any reduction order $>$ and the ground terms $p, q$. However, we assume beforehand that the basic Knuth-Bendix completion procedure or the goal-directed completion procedure or the nonfailing Knuth-Bendix completion procedure will stop on $E,>$, and $p, q$, and estimate its running time. We base our time estimate on the size of the input and the experimental results by the various implementations $[7,9,12,20]$ of all versions of the Knuth-Bendix completion procedure on inputs of similar size. Then we carry out the following steps. Simultaneously, we start all implementations of all versions of the Knuth-Bendix completion procedure on $E$ and $p, q$. We wait for the estimated running time. If none of the procedures stop within this time, then they do not stop at all, or we underestimated the running time. Then we start the procedure $\mathrm{PRO}_{3}$ or $\mathrm{PRO}_{4}$ depending on whether TES E is variable preserving. In some cases $\mathrm{PRO}_{3}$ or $\mathrm{PRO}_{4}$ might give an answer sooner than all implementations of all versions of the Knuth-Bendix completion procedure.

We presented ad hoc examples when procedure $\mathrm{PRO} 3$ was probably more ef- 
ficient than the goal-directed completion procedure [13, 14]. However, to justify the introduction of procedures $\mathrm{PRO}_{3}$ and $\mathrm{PRO}_{4}$, we need further evidence for the efficiency of the procedures $P R O 3$ and $P R O 4$. We should present implementation results and theoretical arguments. We now raise questions on the efficiency of $\mathrm{PRO} 3$ and $\mathrm{PRO}_{4}$ compared to the various versions of the Knuth-Bendix completion procedure.

Question 1. Is it true that for most instances of the ground word problem of a TES E, a correctly chosen version of the Knuth-Bendix completion procedure is more efficient than $\mathrm{PRO} 3$ or $\mathrm{PRO}_{4}$ ?

Question 2. For which instances of the ground word problem of a TES E, is a correctly chosen version of the Knuth-Bendix completion procedure more efficient than $\mathrm{PRO} 3$ or PRO4?

Question 3. Is it decidable for an instance of the ground word problem of a TES $E$, whether a correctly chosen version of the Knuth-Bendix completion procedure is more efficient than $\mathrm{PRO} 3$ or $\mathrm{PRO}_{4}$ ?

Question 4. Is there an instance of the ground word problem of a TES E, such that no version of the Knuth-Bendix completion procedure halts, and PRO3 or PRO4 halts?

We can reduce an instance of the word problem for a TES $E$ to an instance of the ground word problem for $E$ over a larger alphabet $\Delta$. Let $E$ be a TES and $p, q$ arbitrary terms over a ranked alphabet $\Sigma$. Assume that exactly the variables $x_{1}, \ldots, x_{m}$ appear in $p$ or $q$. We now define the ranked alphabet $\Delta$. It contains each element of $\Sigma$. Furthermore, for each $i=1, \ldots, m$, we add a new constant $\#_{i}$ to $\Delta$. We define $p^{\prime}$ from $p$ and $q^{\prime}$ from $q$ by replacing each occurrence of $x_{i}$ with $\#_{i}$ for $i=1, \ldots, m$. Then $p \leftrightarrow_{E}^{*} q$ over $\Sigma$ if and only if $p^{\prime} \leftrightarrow_{E}^{*} q^{\prime}$ over $\Delta$. Thus if we can decide whether $p^{\prime} \leftrightarrow{ }_{E}^{*} q^{\prime}$ over $\Delta$, then we can also decide whether $p \leftrightarrow_{E}^{*} q$ over $\Sigma$.

\section{References}

[1] F. Baader and T. Nipkow Term Rewriting and All That, Cambridge University Press, Cambridge, United Kingdom, 1998.

[2] L. Bachmair, N. Dershowitz, D. A. Plaisted, Completion without failure. In Resolution of equations in algebraic structures, Vol. 2, Rewriting techniques. Edited by H. Ait-Kaci and M. Nivat. pp. 130, Academic Press, Boston, MA, 1989 ,

[3] G. C. Ceitin: Associative calculus with an unsolvable equivalence problem. Tr. Mat. Inst. Akad. Nauk 52, 172-189 (1958) (in Russian).

[4] P. J. Downey, R. Sethi, and R. E. Tarjan: Variations on the Common Subexpression Problem. Journal of the ACM, 27 (1980) 758-771. 
[5] Z. Fülöp and S. Vágvölgyi, Ground term rewriting rules for the word problem of ground term equations, Bulletin of the EATCS, 45 (1991) 186-201.

[6] Z. Fülöp and S. Vágvölgyi, Minimal Equational Representations of Recognizable Tree Languages, Acta Informatica, 34 (1997) 59-84.

[7] J.-M. Gaillourdet, T. Hillenbrand, B. Löchner, H. Spies: The New WALDMEISTER Loop at Work, in Franz Baader (Ed.): Automated Deduction CADE-19, 19th International Conference on Automated Deduction, Proceedings. Lecture Notes in Computer Science 2741 Springer 2003, I 317-321.

[8] J. Gallier, P. Narendran, D. Plaisted, S. Raatz, and W. Snyder, An Algorithm for Finding Canonical Sets of Ground Rewrite Rules in Polynomial Time, Journal of the Association for Computing Machinery, 40 (1993) 1-16.

[9] T. Hillenbrand, Citius altius fortius: Lessons learned from the Theorem Prover WALDMEISTER, Electronic Notes in Theoretical Computer Science 86(1) (2003).

[10] M. Jantzen, Confluent string rewriting, Springer Verlag, Berlin 1988.

[11] Y. Matiyasevich, G. Sénizergues, Decision Problems for Semi-Thue Systems with a Few Rules, Proceedings, 11th Annual IEEE Symposium on Logic in Computer Science, New Brunswick, New Jersey, 27-30 July 1996. IEEE Computer Society Press, 523-531.

[12] Serge Mechveliani, From a Computer Algebra Library to a System with an Equational Prover, in Bruno Buchberger, John A. Campbell (Eds.): Artificial Intelligence and Symbolic Computation, 7th International Conference, AISC 2004, Linz, Austria, September 22-24, 2004, Proceedings. Lecture Notes in Computer Science 3249 Springer 2004, 281-284.

[13] C. Lynch, Goal-Directed Completion Using SOUR Graphs, in Hubert Comon (Ed.): Rewriting Techniques and Applications, 8th International Conference, RTA-97, Proceedings. Lecture Notes in Computer Science 1232 Springer 1997, $8-22$.

[14] C. Lynch, P. Strogova, SOUR graphs for efficient completion, Discrete Mathematics \& Theoretical Computer Science 2 (1998) 1-25.

[15] ] G. Nelson, D. C. Oppen: Fast Decision Procedures Based on Congruence Closure. J. ACM 27 (1980) 356-364

[16] D. Plaisted and A. Sattler-Klein, Proof lengths for equational completion, Information and Computation, 125 (1996) 154-170.

[17] S. Vágvölgyi, A fast algorithm for constructing a tree automaton recognizing a congruential tree language, Theoret. Comput. Sci, 115 (1993) 391-399. 
[18] S. Vágvölgyi, Ground term rewriting. Bull. Eur. Assoc. Theor. Comput. Sci. EATCS No. 102 (2010), 153190.

[19] W. Snyder, A Fast Algorithm for Generating Reduced Ground Rewriting Systems from a set of Ground Equations, Journal of Symbolic Computation, 15 (1993) 415-450.

[20] I. Wehrman, A. Stump, E. M. Westbrook: Slothrop: Knuth-Bendix Completion with a Modern Termination Checker, Frank Pfenning (Ed.): Term Rewriting and Applications, 17th International Conference, RTA 2006, Proceedings. Lecture Notes in Computer Science 4098 Springer 2006, 287-296.

Received 5th June 2015 